Old Dominion University ODU Digital Commons

2012

\title{
Wavelet Collocation Method and Multilevel Augmentation Method for Hammerstein Equations
}

\author{
Hideaki Kaneko \\ Old Dominion University, hkaneko@odu.edu \\ Khomsan Neamprem \\ Boriboon Novaprateep
}

Follow this and additional works at: https://digitalcommons.odu.edu/mathstat_fac_pubs

Part of the Numerical Analysis and Computation Commons

\section{Repository Citation}

Kaneko, Hideaki; Neamprem, Khomsan; and Novaprateep, Boriboon, "Wavelet Collocation Method and Multilevel Augmentation Method for Hammerstein Equations" (2012). Mathematics \& Statistics Faculty Publications. 1.

https://digitalcommons.odu.edu/mathstat_fac_pubs/1 


\title{
WAVELET COLLOCATION METHOD AND MULTILEVEL AUGMENTATION METHOD FOR HAMMERSTEIN EQUATIONS*
}

\author{
HIDEAKI KANEKO ${ }^{\dagger}, \mathrm{KHOMSAN} \mathrm{NEAMPREM}^{\ddagger}$, AND BORIBOON NOVAPRATEEP§
}

\begin{abstract}
A wavelet collocation method for nonlinear Hammerstein equations is formulated. A sparsity in the Jacobian matrix is obtained which gives rise to a fast algorithm for nonlinear solvers such as the Newton's method and the quasi-Newton method. A fast multilevel augmentation method is developed on a transformed nonlinear equation which gives an additional saving in computational time.
\end{abstract} method

Key words. wavelet collocation method, Hammerstein equations, fast multilevel augmentation

AMS subject classifications. 45G05, 45G10, 47H30, 65R20

DOI. $10.1137 / 100809246$

1. Introduction. Hammerstein equations represent an integral reformulation of nonlinear boundary value problems. There have been a number of papers written in recent years which establish numerical techniques for finding an approximation of a solution of the Hammerstein equation (see, e.g., [8], [13], [14], [15], [16], [17], [18], [19]). Generally, numerical solutions are found by solving a system of nonlinear equations using the Newton's method, the secant method, or the quasi-Newton method. In executing its iterative process, the Newton's method as well as the secant method require at each step of their iterations updating of the Jacobian matrix, which is normally dense. The quasi-Newton method eliminates the need for this update by fixing the Jacobian matrix. This results in a significant reduction in the overall cost of computation, even though convergence may be slower. This reduction in computational cost arises because a large portion of the CPU time is used for assembling of the Jacobian matrices. We point out that the Jacobian matrices used in the Newton's method, the secant method, and the quasi-Newton method are dense when spline bases are used.

In general, advancing one iteration in the Newton's method or in the quasi-Newton method requires $\mathcal{O}\left(N^{2}\right)$ operation counts in an $N \times N$ system, and thus if one desires a higher accuracy in approximation, computational complexity increases with $N$. In [8], a class of wavelets in conjunction with a fast multilevel augmentation method was used to approximate a solution of the Hammerstein equation which uses a fast multilevel augmentation method. The method is a generalization of the multilevel method for linear operator equations which was first established in [5]. It was shown that when it is applied to the Hammerstein equation, the complexity of computation

*Submitted to the journal's Methods and Algorithms for Scientific Computing section September 21, 2010; accepted for publication (in revised form) October 26, 2011; published electronically February 7, 2012. This research is supported by the Centre of Excellence in Mathematics, the Commission on Higher Education, Thailand.

http://www.siam.org/journals/sisc/34-1/80924.html

$\dagger$ Department of Mathematics and Statistics, Old Dominion University, Norfolk, VA 23529-0077 (hkaneko@odu.edu).

${ }^{\ddagger}$ Department of Mathematics, Faculty of Applied Science, King Mongkut’s University of Technology North Bangkok, Bangkok 10800, Thailand (khomsann@kmutnb.ac.th), and the Centre of Excellence in Mathematics, CHE, Si Ayutthaya Rd., Bangkok 10400, Thailand.

$\S$ Department of Mathematics, Faculty of Science, Mahidol University, Bangkok 10400, Thailand (scbnv@mahidol.ac.th), and the Centre of Excellence in Mathematics, CHE, Si Ayutthaya Rd., Bangkok 10400, Thailand. 
decreases to $\mathcal{O}(N \log N)$. The multilevel method has the advantage of a multiresolution structure of a wavelet basis and finds a solution with higher accuracy by augmenting a solution from a lower resolution level by adding correction terms. The technique was successfully implemented to approximate the solution of the Fredholm second kind integral equations in [11] and was formulated in general terms for the second kind operator equations in [10].

It was established in [1] that a class of wavelet bases can be applied to approximate solutions of the Fredholm integral equations of the second kind, which result in a sparse linear system. This result has found a significant implication in the reduction of an overall computational expense in approximating the solution of the Fredholm equation since, as stated earlier, a standard spline basis results in a linear system which is dense. This discovery was greatly expanded and generalized in recent years in a series of papers, [21], [23], [7], [9], where truncation strategies were employed to produce sparse systems, leading to fast algorithms. The latter three papers deal with the wavelet collocation methods for the Fredholm integral equations of the second kind. One of the goals of our paper is to extend those methods to formulate a wavelet collocation method for Hammerstein equations. The sparsity is generated by the two key properties of the wavelets. First, wavelets and their functionals have vanishing moments, and second, it is possible to retain only those components in the matrix of coefficients associated with wavelets whose supports are "separated from the diagonal," the concept introduced in [1] and expanded in [21], [23], [7], [9]. There are $\mathcal{O}(N \log N)$ number of supports which are separated from the diagonal, and this in turn produces the sparsity in the resulting linear system. When an appropriate truncation strategy is employed, it produces a fast algorithm amenable to generating an approximation of the optimal order of accuracy. The main difficulty in extending the methods established in [7], [9] to nonlinear Hammerstein equations lies in the fact that the solution appears not directly under the integral term but rather under a nonlinear term. This prevents the application of the vanishing moments of the wavelets and their corresponding linear functionals when the kernel of the integral operator is expanded in the Taylor series. In this paper, we show in section 3 that by "linearizing" the Hammerstein equation, many of the important techniques and results established in [7], [9] can be restored to formulate the current wavelet collocation method for the nonlinear equation. The method produces Jacobian matrices which are sparse. This generalizes a similar result for the Petrov-Galerkin method for the Hammerstein equation [12].

In section 4, a multilevel augmentation method applied directly to the linearized Hammerstein equation is discussed. The new fast multilevel augmentation method is similar to the one established in [8] but also takes advantage of the sparse structure of the Jacobian matrix, which leads to an additional reduction in the overall computational cost. In order to make this paper as self-contained as possible, section 2 is used to review some existing results necessary to establish the new results in this paper. Here, we quote much from [7] and [9].

2. Preliminaries. In this section, we review necessary preliminary information which will be used to establish the wavelet collocation method for the nonlinear Hammerstein equation in section 3 .

2.1. A wavelet collocation method for Fredholm integral equations of the second kind. For $d \geq 1$, we assume that $E \subseteq \mathbf{R}^{d}$ is compact and first consider 
the second kind weakly singular Fredholm equations

$$
u(t)-\int_{E} k(t, s) u(s) d s=f(t), \quad t \in E,
$$

where $k, f \in C(E)$, and $\psi$ are known functions and $u$ is the function to be determined. Denote

$$
K u(t):=\int_{E} k(s, t) u(s) d s,
$$

and assume that $K: C(E) \rightarrow C(E)$ is a compact operator. Equation (2.1) can be written as

$$
(I-K) u=f .
$$

In [6], Chen, Micchelli, and Xu introduced a class of multiscale piecewise polynomial functions and corresponding multiscale collocation functionals. These multiscale functions and functionals form a foundation to formulate the wavelet collocation method for the Fredholm integral equation of the second kind (2.3) [7], [9]. A consequence of the wavelet collocation method is that its multiresolution structure provides a truncation strategy which is amenable to a fast algorithm. This provides an alternative numerical method to the wavelet Galerkin method [21] in which a different truncation strategy had been developed to generate a sparse linear system for (2.3). In what follows in this section, we summarize results from [6], [7], [8], [9] and assume that $E=[0,1]$. To understand the wavelet collocation method, we first introduce a family of contractive mappings $\Phi_{\mu}:=\left\{\phi_{e}: e \in Z_{\mu}\right\}, Z_{\mu}:=\{0,1, \ldots, \mu-1\}$, with a fixed positive integer $\mu$ on the interval $I:=[0,1]$ defined by

$$
\phi_{e}(t)=\frac{t+e}{\mu}, \quad t \in I, \quad e \in Z_{\mu},
$$

where

$$
I=\bigcup_{e \in Z_{\mu}} \phi_{e}(I), \text { and meas }\left\{\phi_{e}(I) \cap \phi_{e^{\prime}}(I)\right\}=0, \quad e \neq e^{\prime} .
$$

Let $\mathbf{X}_{0}$ be the space of polynomials of order $\kappa>0$ on $I$ and $\mathcal{T}_{e}: L^{\infty}(I) \rightarrow L^{\infty}(I)$ linear operators which are defined as

$$
\mathcal{T}_{e} x:=x \circ \phi_{e}^{-1} \chi_{\phi_{e}(I)} \quad \text { for } x \in L^{\infty}(I),
$$

where $\chi_{A}$ is the characteristic function of $A$ for a subset $A \subset I$. The process described in (2.4) underpins the fact that the subspaces $\mathbf{X}_{n}$ defined below are comprised of the scaled versions of the functions in $\mathbf{X}_{0}$. This provides a basis for the multiresolution decomposition of $\mathbf{X}_{n}$ described in (2.5). It should be pointed out that, if one desires to construct a family of piecewise polynomials which are orthonormal in $L^{2}(I)$, an orthonormal basis for $\mathbf{X}_{0}$ must be chosen. Such a basis was used in the formulation of the fast wavelet Galerkin method in [21]. For the current problem, we select a basis for $\mathbf{X}_{0}$ which is interpolatory. A sequence of subspaces $\mathbf{X}_{n}$ can be defined by using operator (2.4) as follows:

$$
\mathbf{X}_{n+1}=\bigoplus_{e \in Z_{\mu}} \mathcal{T}_{e} \mathbf{X}_{n}, \quad n \in \mathbf{N}_{0}
$$


where $\mathbf{N}_{0}:=\{0,1, \ldots\}$ and $\mathbf{A} \oplus \mathbf{B}$ denotes a direct sum of spaces $\mathbf{A}$ and $\mathbf{B}$. It is easy to see that the following statements are true:

(P1) The sequence of the subspaces is nested, i.e., $\mathbf{X}_{n} \subseteq \mathbf{X}_{n+1}, n \in \mathbf{N}_{0}$.

(P2) $\mathbf{X}_{n}$ is the space of piecewise polynomials of order $\kappa$ on $I$ with knots at $j / \mu^{n}, j=1,2, \ldots, \mu^{n}-1$.

(P3) $\operatorname{dim}\left(\mathbf{X}_{n}\right)=\kappa \mu^{n}, n \in \mathbf{N}_{0}$.

Because the subspaces $\mathbf{X}_{n}$ have the nested property, we can obtain

$$
\mathbf{X}_{n}=\mathbf{W}_{0} \oplus \mathbf{W}_{1} \oplus \cdots \oplus \mathbf{W}_{n},
$$

with $\mathbf{W}_{0}:=\mathbf{X}_{0}$, and $\mathbf{W}_{i}$ is the orthogonal complement of $\mathbf{X}_{i-1}$ in $\mathbf{X}_{i}, i=1,2, \ldots, n$, i.e.,

$$
\mathbf{X}_{i}=\mathbf{X}_{i-1} \oplus \mathbf{W}_{i}
$$

It is known [20] that

$$
\mathbf{W}_{n+1}=\bigoplus_{e \in Z_{\mu}} \mathcal{T}_{e} \mathbf{W}_{n}, \quad n \in \mathbf{N}
$$

when the initial space $\mathbf{W}_{1}$ is constructed. For $i \in \mathbf{N}_{0}$, with $\omega(i):=\operatorname{dim}\left(\mathbf{W}_{i}\right)$, we have

$$
\omega(i)=\kappa(\mu-1) \mu^{i-1},
$$

and $\left\{\mathbf{W}_{i}\right\}_{i=0}^{n}$ serve as multiscale subspaces of $L^{\infty}(I)$. Next, wavelet bases and corresponding collocation functionals on $L^{\infty}(I)$ must be constructed. To define a basis for $\mathbf{W}_{1}$, we use the fact that $\mathbf{W}_{1}$ is a subspace of $\mathbf{X}_{1}$, and every element $w \in \mathbf{W}_{1}$ must be orthogonal to $\mathbf{X}_{0}=\mathbf{W}_{0}$, i.e.,

$$
\left(w, w_{0 j}\right)=0, \quad j \in Z_{\kappa},
$$

where $\left\{w_{0 j}, j \in Z_{\kappa}\right\}$ is a basis for $\mathbf{W}_{0}$ and $\left(w, w_{0 j}\right):=\int_{E} w(s) w_{0 j}(s) d s$. Note that the dimension of $\mathbf{X}_{1}$ is $\kappa \mu$, and (2.6) gives $r:=\kappa(\mu-1)$ linearly independent solutions $w_{1 j}, j \in Z_{r}$, which form a basis for $\mathbf{W}_{1}$. To generate bases for the subsequent wavelet spaces $\mathbf{W}_{i}, i>1$, we define a composition operator $\mathcal{T}_{\mathbf{e}}$ by

$$
\mathcal{T}_{\mathbf{e}}:=\mathcal{T}_{e_{0}} \circ \cdots \circ \mathcal{T}_{e_{i-1}},
$$

where

$$
\mathbf{e}:=\left(e_{0}, \ldots, e_{i-1}\right) \in Z_{\mu}^{i}:=\underbrace{Z_{\mu} \times \cdots \times Z_{\mu}}_{i \text { times }}, e_{j} \in Z_{\mu} \text { for } j=0, \ldots, i-1 .
$$

With

$$
\mu(\mathbf{e}):=\mu^{(i-1)} e_{0}+\cdots+\mu e_{i-2}+e_{i-1},
$$

and for $i=2,3, \ldots, n$, we let

$$
w_{i j}:=\mathcal{T}_{\mathbf{e}} w_{1 l}, \quad j=\mu(\mathbf{e}) r+l, \quad \mathbf{e} \in Z_{\mu}^{i-1}, \quad l \in Z_{r} ;
$$

then $\mathbf{W}_{i}=\operatorname{span}\left\{w_{i j}: j \in Z_{\omega(i)}\right\}$.

In order to facilitate the establishment of a wavelet collocation method, Chen, Micchelli, and $\mathrm{Xu}$ [6], [7] defined the notion of a refinable set and consequently the 
collocation functionals in $\mathbf{V}^{*}$ with $\mathbf{V}:=C(I)$. Now select $\kappa$ distinct points $V_{0}:=\left\{t_{j}\right.$ : $\left.j \in Z_{\kappa}\right\}$ in $I$ so that $V_{0}$ is refinable related to the mappings $\Phi_{\mu}$, i.e., $V_{0} \subseteq V_{1}:=\Phi_{\mu}\left(V_{0}\right)$. For example, it is shown in [6] that, when $\mu$ and $\kappa+1$ are relatively prime, the set

$$
V_{0}:=\left\{\frac{j+1}{\kappa+1}: j \in Z_{\kappa}\right\}
$$

is refinable relative to $\Phi_{\mu}$. For every point $t_{j} \in V_{0}$, we define $\ell_{0 j}:=\delta_{t_{j}}, j \in Z_{\kappa}$, where $\left\langle\delta_{t_{j}}, x\right\rangle:=x\left(t_{j}\right)$ with $x \in C(I)$. We order the points in $V_{1}$ so that the first $\kappa$ points coincide with the points in the set $V_{0}$. For each $j \in Z_{\kappa(\mu-1)}$, we find the vector $\left[c_{j s}: s \in Z_{\kappa \mu}\right]$ such that

$$
\ell_{1 j}:=\sum_{s \in Z_{\kappa \mu}} c_{j s} \delta_{t_{s}}, \quad j \in Z_{\kappa(\mu-1)}
$$

satisfy equations

$$
\left\langle\ell_{1 j}, w_{0 j^{\prime}}\right\rangle=0, \quad j^{\prime} \in Z_{\kappa},
$$

and

$$
\left\langle\ell_{1 j}, w_{1 j^{\prime}}\right\rangle=\delta_{j j^{\prime}}, \quad j^{\prime} \in Z_{r} .
$$

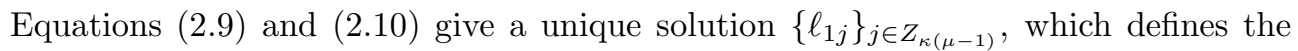
interpolatory linear functionals $\ell_{1 j}$ relative to the basis function in $\mathbf{W}_{1}$. To define linear functionals with the interpolation property relative to the basis $\left\{w_{i j}\right\}, i>1$, we let $\mathcal{L}_{e}: \mathbf{V}^{*} \rightarrow \mathbf{V}^{*}$ be defined by the equation

$$
\left\langle\mathcal{L}_{e} \ell, x\right\rangle=\left\langle\ell, x \cdot \phi_{e}\right\rangle \quad \text { for all } x \in \mathbf{V} \text { and } \ell \in \mathbf{V}^{*},
$$

and for $\mathbf{e}:=\left(e_{0}, \ldots, e_{n-1}\right) \in Z_{\mu}^{n}$,

$$
\mathcal{L}_{\mathrm{e}}:=\mathcal{L}_{e_{0}} \circ \cdots \circ \mathcal{L}_{e_{n-1}} .
$$

Finally, for $i=2,3, \ldots, n$,

$$
\ell_{i j}:=\mathcal{L}_{\mathrm{e}} \ell_{1 l}, \quad j=\mu(\mathbf{e}) r+l, \quad \mathbf{e} \in Z_{\mu}^{i-1}, \quad l \in Z_{r} .
$$

The basis functions $w_{i j}$ and the collocation functional $\ell_{i j}$ which are generated by using (2.11) and (2.12) have the following important properties:

(P4) They have vanishing moments of order $\kappa$; that is, for all elements $p$ in the space of polynomials of degree less than $\kappa$ on $I$,

$$
\left\langle\ell_{i j}, p\right\rangle=0, \quad\left(w_{i j}, p\right)=0, \quad j \in Z_{\kappa(\mu-1) \mu^{i-1}}, \quad j \in \mathbf{N} .
$$

(P5) They satisfy the semibiorthogonality; that is, for any $i, i^{\prime} \in \mathbf{N}_{0}$,

$$
\left\langle\ell_{i^{\prime} j^{\prime}}, w_{i j}\right\rangle=\delta_{i i^{\prime}} \delta_{j j^{\prime}}, \quad(i, j),\left(i^{\prime}, j^{\prime}\right) \in \mathbf{U}, \quad i \leq i^{\prime},
$$

where $\mathbf{U}:=\left\{(i, j): i \in \mathbf{N}_{0}, j \in Z_{\omega(i)}\right\}$.

(P6) There exists a positive constant $\gamma$ for which

$$
\sum_{j \in Z_{w(i)}}\left|\left\langle\ell_{i^{\prime} j^{\prime}}, w_{i j}\right\rangle\right| \leq \gamma, \quad(i, j),\left(i^{\prime}, j^{\prime}\right) \in \mathbf{U}, \quad i>i^{\prime} .
$$

Copyright $@$ by SIAM. Unauthorized reproduction of this article is prohibited. 
The functionals $\ell_{i j}$ belong to $X^{*}$, the dual space of $X=L^{\infty}(I)$ with corresponding interpolation functions $w_{i j} \in X$. Here, using the Hahn-Banach theorem, we may assume $X^{*}$ to be the dual of $C(I)$; see [4], [7]. Let $U_{n}:=\left\{(i, j): j \in Z_{\omega(i)}, i \in Z_{n+1}\right\}$ and $\mathbf{u}_{n}:=\left[u_{i j}:(i, j) \in U_{n}\right]^{T}$. We let

$$
\mathbf{L}_{0}=\operatorname{span}\left\{\ell_{0 j}: j \in Z_{\omega(0)}\right\}, \quad \mathbf{V}_{i}=\operatorname{span}\left\{\ell_{i j}: j \in Z_{\omega(i)}\right\}, \quad i>0 .
$$

Since $\mathbf{X}_{n}=\operatorname{span}\left\{w_{i j}:(i, j) \in U_{n}\right\}$, analogously we define

$$
\mathbf{L}_{n}=\operatorname{span}\left\{\ell_{i j}:(i, j) \in U_{n}\right\}, \quad n>\mathbf{N}_{0} .
$$

This implies the decomposition

$$
\mathbf{L}_{n+1}=\mathbf{L}_{n} \oplus \mathbf{V}_{n+1}
$$

and

$$
\mathbf{L}_{k+m}=\mathbf{L}_{k} \oplus \mathbf{V}_{k, m},
$$

where $\mathbf{V}_{k, m}=\mathbf{V}_{k+1} \oplus \cdots \oplus \mathbf{V}_{k+m}$.

Examples of piecewise linear, quadratic and cubic polynomials and their corresponding collocation functionals with multilevel structure can be found in [9]. They can also be found in [6] and [8]. We include the linear case for illustration and for the reader's convenience, since the linear basis is used for numerical examples reported in sections 3 and 4 .

2.2. Piecewise linear wavelet basis. Here $\kappa=2$ and $\mu=2$, and we choose the knots $t_{j}=\frac{j}{2^{n}}, j=1,, 2, \ldots, 2^{n}-1 . \Phi_{2}=\left\{\phi_{0}, \phi_{1}\right\}$ is the family of contractions $\phi_{e}(t)=\frac{t+e}{2}, e \in Z_{2} . G_{0}=\left\{\frac{1}{3}, \frac{2}{3}\right\}$ is refinable relative to $\Phi_{2}$ and $G_{1}=\Phi_{2}\left(G_{0}\right)=$ $\left\{\frac{1}{6}, \frac{1}{3}, \frac{2}{3}, \frac{5}{6}\right\}$. Let $\mathbf{X}_{0}=\mathbf{W}_{0}=\operatorname{span}\left\{w_{00}, w_{01}\right\}$, where

$$
w_{00}(t)=2-3 t, \quad w_{01}(t)=-1+3 t, \quad t \in I .
$$

A basis for $\mathbf{W}_{1}$ is given by

$$
w_{10}(t)=\left\{\begin{array}{ll}
1-\frac{9}{2} t, & t \in\left[0, \frac{1}{2}\right], \\
-1+\frac{3}{2} t, & t \in\left(\frac{1}{2}, 1\right],
\end{array} \quad w_{11}(t)= \begin{cases}\frac{1}{2}-\frac{3}{2} t, & t \in\left[0, \frac{1}{2}\right], \\
-\frac{7}{2}+\frac{9}{2} t, & t \in\left(\frac{1}{2}, 1\right] .\end{cases}\right.
$$

The corresponding collocation functionals are given by

$$
\ell_{00}=\delta_{\frac{1}{3}}, \quad \ell_{01}=\delta_{\frac{2}{3}}, \quad \ell_{10}=\delta_{\frac{1}{6}}-\frac{3}{2} \delta_{\frac{1}{3}}+\frac{1}{2} \delta_{\frac{2}{3}}, \quad \ell_{11}=\frac{1}{2} \delta_{\frac{1}{3}}-\frac{3}{2} \delta_{\frac{2}{3}}+\delta_{\frac{5}{6}} .
$$

Figures 1-6 illustrate the linear multiscale wavelets and the corresponding collocation functionals shown below.

The wavelet collocation method approximates the solution $u$ of $(2.3)$ by $u_{n}:=$ $\sum_{(i, j) \in U_{n}} u_{i j} w_{i j}$, where

$$
\left\langle\ell_{i^{\prime} j^{\prime}}, u_{n}-K u_{n}\right\rangle=\left\langle\ell_{i^{\prime} j^{\prime}}, f\right\rangle, \quad\left(i^{\prime}, j^{\prime}\right) \in U_{n} .
$$

Equivalently, we solve

$$
\left(\mathbf{E}_{n}-\mathbf{K}_{n}\right) \mathbf{u}_{n}=\mathbf{f}_{n},
$$

where

$$
\begin{aligned}
\mathbf{E}_{n} & :=\left[\left\langle\ell_{i^{\prime} j^{\prime}}, w_{i j}\right\rangle:\left(i^{\prime}, j^{\prime}\right),(i, j) \in U_{n}\right], \\
\mathbf{K}_{n} & :=\left[\left\langle\ell_{i^{\prime} j^{\prime}}, K w_{i j}\right\rangle:\left(i^{\prime}, j^{\prime}\right),(i, j) \in U_{n}\right],
\end{aligned}
$$

and $\mathbf{f}_{n}:=\left[\left\langle\ell_{i^{\prime} j^{\prime}}, f\right\rangle:\left(i^{\prime}, j^{\prime}\right) \in U_{n}\right]^{T}$. 

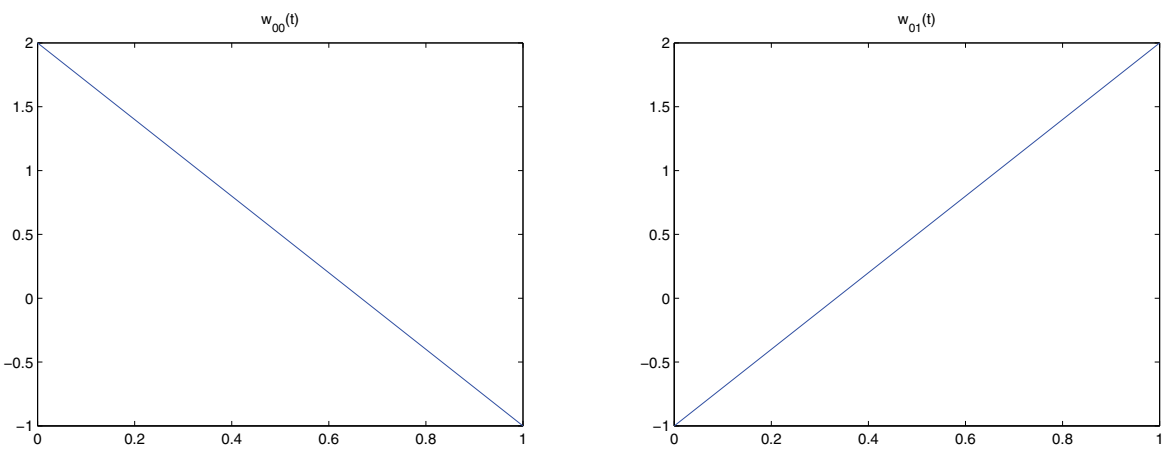

Fig. 1. Linear multiscale wavelet bases on $\mathbf{W}_{0}$.
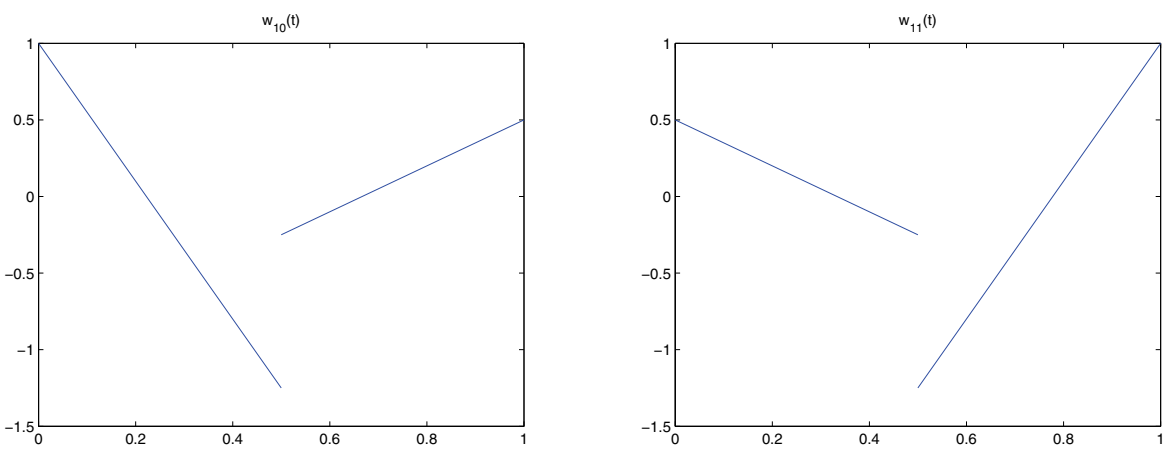

FIG. 2. Linear multiscale wavelet bases on $\mathbf{W}_{1}$.
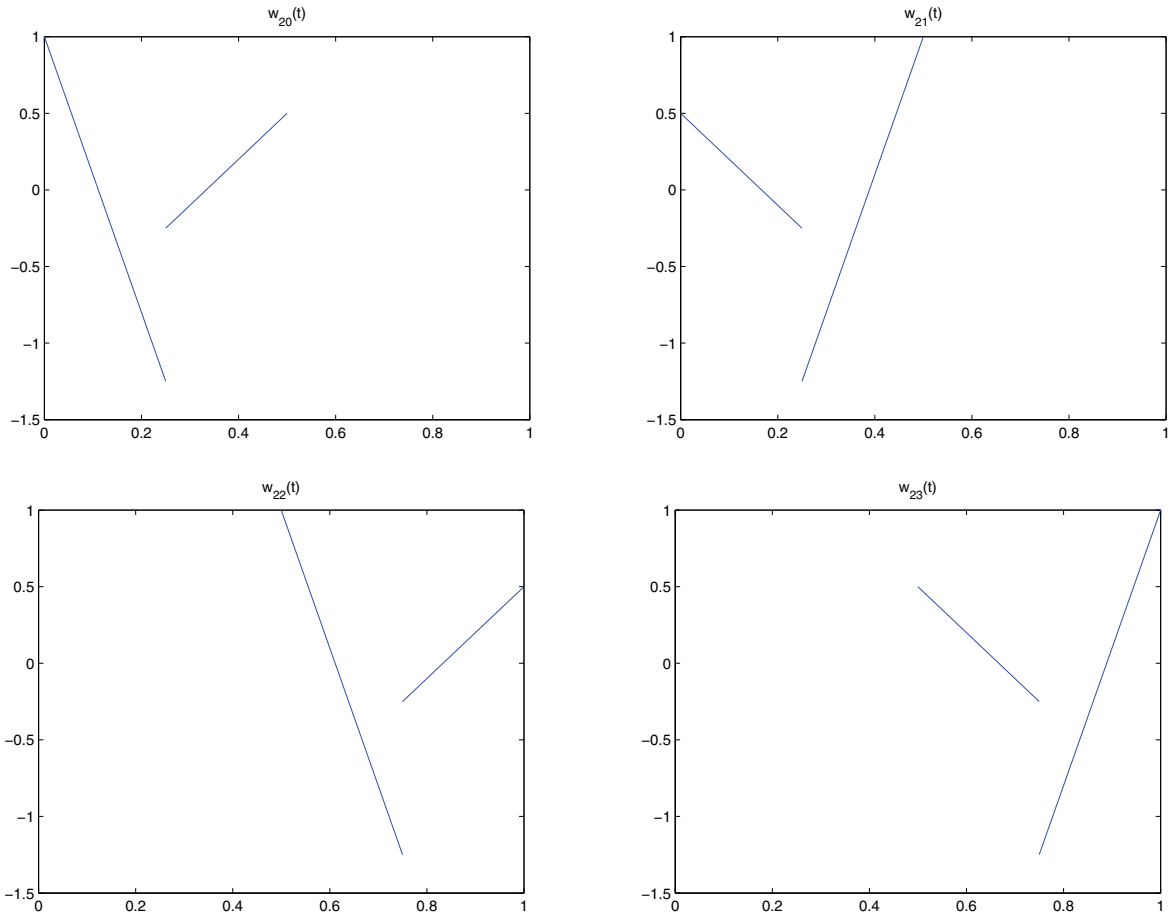

FIG. 3. Linear multiscale wavelet bases on $\mathbf{W}_{2}$.

Copyright (c) by SIAM. Unauthorized reproduction of this article is prohibited. 

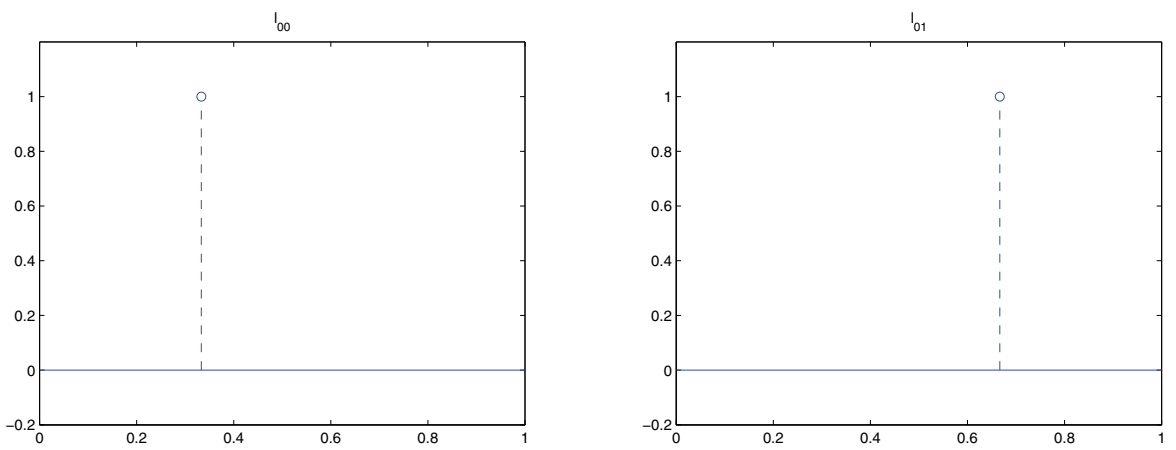

FIG. 4. Linear multiscale collocation functionals associated with $\mathbf{W}_{0}$.
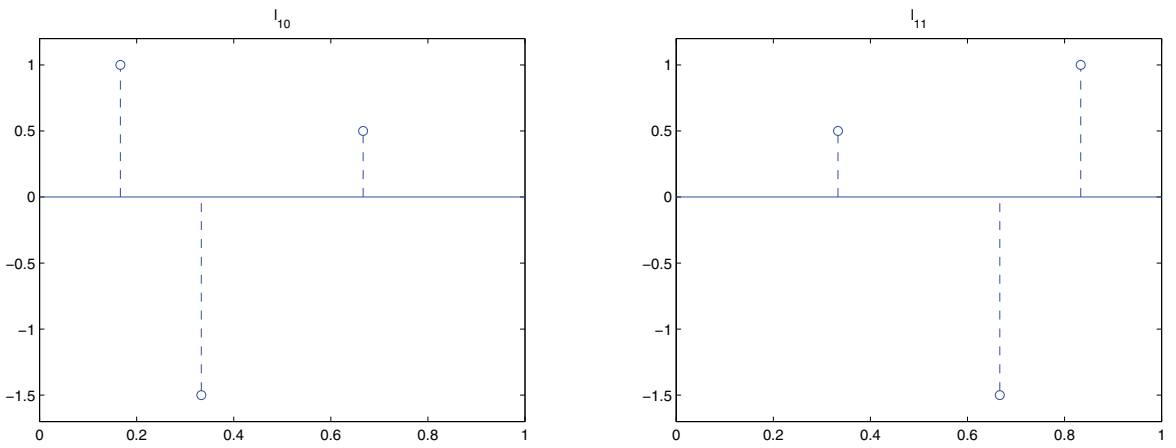

FIG. 5. Linear multiscale collocation functionals associated with $\mathbf{W}_{1}$.
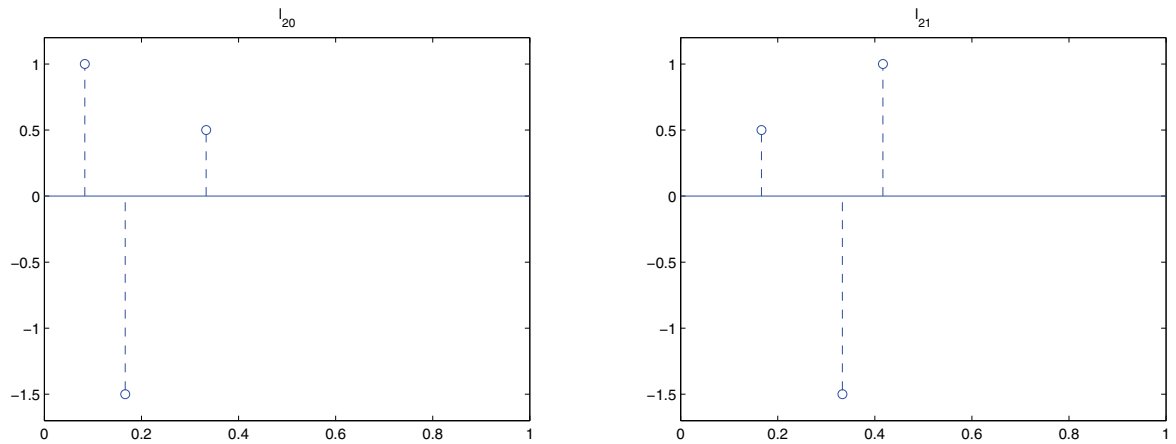

$\mathrm{I}_{22}$
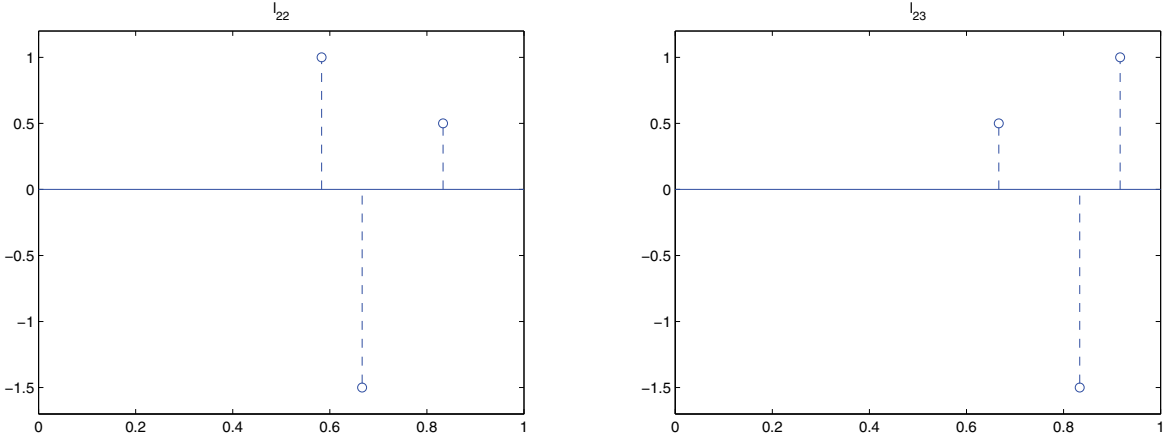

FIG. 6. Linear multiscale collocation functionals associated with $\mathbf{W}_{2}$.

Copyright (c) by SIAM. Unauthorized reproduction of this article is prohibited. 
2.3. Truncation strategy. It is assumed in [7], [9] that the kernel $k(t, s)$ has a weak singularity along the diagonal $s=t$, i.e., $k(t, s)$ has continuous partial derivatives $D_{t}^{\alpha} D_{s}^{\beta} k(t, s)$ for $\alpha \leq \kappa$ and $\beta \leq \kappa$ when $t, s \in I$ and $s \neq t$ and there exist positive constants $\sigma<1$ and $\theta$ such that

$$
\left|D_{t}^{\alpha} D_{s}^{\beta} k(t, s)\right| \leq \frac{\theta}{|t-s|^{\sigma+2 \kappa}} .
$$

The truncation strategies, which engender fast wavelet collocation algorithms to approximate the solution $\mathbf{u}_{n}$ of (2.16), are built upon the vanishing moment properties (2.13), an analysis on how the supports of $\ell_{i^{\prime} j^{\prime}}$ and $w_{i j}$ are separated from the diagonal, and the property of the kernel described in (2.19). Let $K_{i^{\prime} j^{\prime}, i j}=\left\langle\ell_{i^{\prime} j^{\prime}}, K w_{i j}\right\rangle$ and $\hat{S}_{i j}$ and $S_{i j}$ denote the supports of $\ell_{i^{\prime} j^{\prime}}$ and $w_{i j}$, respectively. Setting $d_{i j}:=\operatorname{diam}\left(S_{i j}\right)$ and $d_{i}=\max \left\{d_{i j}: j \in Z_{\omega(i)}\right\}, i \geq 0$, the size of $K_{i^{\prime} j^{\prime}, i j}$ can be estimated as follows.

Lemma 2.1 (see Lemma 3.1 in [7]). If there exist constant $r>1$ such that

$$
\operatorname{dist}\left(S_{i j}, S_{i^{\prime} j^{\prime}}\right) \geq r\left(d_{i}+d_{i^{\prime}}\right)
$$

then there exist positive constants c such that

$$
\left|K_{i^{\prime} j^{\prime}, i j}\right| \leq c\left(d_{i} d_{i^{\prime}}\right)^{\kappa} \sum_{s \in \hat{S}_{i^{\prime} j^{\prime}}} \int_{S_{i j}} \frac{1}{|s-t|^{2 \kappa+\sigma}} d t,
$$

where $c=\frac{\theta \theta_{1}^{2} e^{\frac{2 d}{1-r^{-1}}}}{\kappa^{2}\left(1-r^{-1}\right)^{\sigma}}$ in which $\theta$ and $\sigma$ are defined in $(2.19)$ and $\left\|\ell_{i j}\right\|+\left\|w_{i j}\right\|_{\infty} \leq \theta_{1}$.

In this paper, as was done in [9], we also denote $\hat{S}_{i j}$ by $S_{i j}$. The truncation strategy in [9] is more practical to implement than the theoretical one developed in [7]. To describe it, let $S_{i j}$ denote the supports of $\ell_{i j}$ and $w_{i j}$. More specifically, with $I_{j, m}:=\left[\frac{j}{m}, \frac{j+1}{m}\right]$ and $(i, j) \in U_{n}$,

$$
S_{i j}=I_{\mu(\mathbf{e}), \mu^{i-1}}
$$

where $\mathbf{e} \in Z_{\mu}^{i-1}$ and $\ell \in Z_{r}, r=k(\mu-1)$ are unique elements such that $j=\mu(\mathbf{e})+\ell$. Now, partition $\mathbf{K}_{n}$ into a block matrix

$$
\mathbf{K}_{n}=\left[\mathbf{K}_{i^{\prime} i}: i^{\prime}, i \in Z_{n+1}\right],
$$

where $\mathbf{K}_{i^{\prime} i}:=\left[K_{i^{\prime} j^{\prime}, i j}: j^{\prime} \in Z_{\omega\left(i^{\prime}\right)}, j \in Z_{\omega(i)}\right]$. Each block is then further partitioned into subblocks classified under the condition that the entries in a same subblock have the same support:

$$
\mathbf{K}_{i^{\prime} i}=\left[\mathbf{K}_{q^{\prime} q}^{i^{\prime} i}: q^{\prime}, q \in Z_{\mu^{i_{0}-1}}\right], \quad \text { where } i_{0}=\min \left\{i^{\prime}, i\right\},
$$

and for $i \geq i^{\prime}$,

$$
\mathbf{K}_{q^{\prime} q}^{i^{\prime} i}:=\left[K_{i^{\prime} j^{\prime}, i j}: j^{\prime}-q^{\prime} r \in Z_{r}, j-q \mu^{i-i^{\prime}} r \in Z_{\mu^{i-i^{\prime}} r}\right],
$$

and for $i<i^{\prime}$,

$$
\mathbf{K}_{q^{\prime} q}^{i^{\prime} i}:=\left[K_{i^{\prime} j^{\prime}, i j}: j^{\prime}-q^{\prime} \mu^{i^{\prime}-i} r \in Z_{\mu^{i^{\prime}-i} r}, j-q r \in Z_{r}\right] .
$$


Finally, a family of positive numbers $p:=\left[p_{i^{\prime} i}: i^{\prime}, i \in Z_{n+1}\right]$ is chosen, and a sparse matrix $\widetilde{\mathbf{K}}_{i^{\prime} i}=\left[\mathbf{K}_{q^{\prime} q}^{i^{\prime} i}(p): q^{\prime}, q \in Z_{\mu^{i_{0}-1}}\right]$ is obtained by the truncation strategy

$$
\mathbf{K}_{q^{\prime} q}^{i^{\prime} i}(p)= \begin{cases}\mathbf{K}_{q^{\prime} q}^{i^{\prime} i}, & \left|q-q^{\prime}\right| \leq p_{i^{\prime} i} \\ 0 & \text { otherwise }\end{cases}
$$

The crux of this method lies in the selection of parameters $p_{i^{\prime} i}$ in a way that the retained subblocks $\mathbf{K}_{q^{\prime} q}^{i^{\prime} i}(p)$ contain all the components $K_{i^{\prime} j^{\prime}, i j}$ which are retained in the theoretical truncation strategy in [7]; thereby the compressed system gives an approximation of optimal accuracy, and the number of nonzero elements is still in the order of $\mathcal{O}(f(n) \log f(n))$ with

$$
f(n):=\operatorname{dim}\left(\mathbf{X}_{n}\right) .
$$

Let $\widetilde{\mathbf{A}}_{n}:=\mathbf{E}_{n}-\widetilde{\mathbf{K}}_{n}$ and choose a constant $b^{\prime}$ such that $\frac{k-\sigma^{\prime}}{2 \kappa-\sigma^{\prime}}<b^{\prime} \leq 1$ with $0<\sigma^{\prime}<$ $1-\sigma$.

Theorem 2.2 (see Theorem 3.3 of [9]). Suppose that $u \in W^{\kappa, \infty}(I)$. For any constants $a>0$ and $\nu>1$, for $i^{\prime}<i$ let

$$
p_{i^{\prime} i}:=1+\max \left\{a \mu^{b^{\prime}\left(n-i^{\prime}\right)-i+i^{\prime}-1}, \nu\left(\mu^{-i+i^{\prime}}+1\right)\right\},
$$

and for $i^{\prime} \geq i$ let

$$
p_{i^{\prime} i}:=1+\max \left\{a \mu^{b^{\prime}\left(n-i^{\prime}\right)-1}, \nu\left(\mu^{-i+i^{\prime}}+1\right)\right\} .
$$

Then there exists a positive constant $c$ such that

$$
\left\|u-\tilde{u}_{n}\right\|_{\infty} \leq c f(n)^{-\kappa} \log ^{\tau} f(n)\|u\|_{k, \infty},
$$

where $\tau=1$ or $2, f(n)=\operatorname{dim}\left(\mathbf{X}_{n}\right),\left(\mathbf{E}_{n}-\widetilde{\mathbf{K}}_{n}\right) \tilde{\mathbf{u}}_{n}=\mathbf{f}_{n}$, and

$$
N\left(\widetilde{\mathbf{A}}_{n}\right)=\mathcal{O}(f(n) \log f(n)),
$$

where $N\left(\widetilde{\mathbf{A}}_{n}\right)$ denotes the number of nonzero entries in the matrix $\widetilde{\mathbf{A}}_{n}$.

3. A wavelet collocation method for Hammerstein equations. We consider the Hammerstein equation which takes the following form:

$$
u(t)-\int_{I} k(t, s) \psi(s, u(s)) d s=f(t), \quad t \in I,
$$

where, for every $s \in I, k(\cdot, s) \in L^{1}(I)$ and we assume first that it is a weakly singular kernel satisfying (2.19) and $f \in L^{\infty}(I)$. The functions $k, f$, and $\psi$ are known and $u$ is the function to be determined. Denote

$$
\Psi(u)(t):=\psi(t, u(t)),
$$

where $K, \Psi: C(I) \rightarrow C(I)$, and $K$, defined in (2.2), is assumed to be a compact operator. Equation (3.1) can be written as

$$
(I-K \Psi) u=f .
$$

We assume throughout the section the following conditions on $k, f$, and $\psi$.

(A1) $\lim _{t \rightarrow \tau}\|k(t, s)-k(\tau, s)\|_{\infty}=0, \tau \in[0,1]$. 
(A2) $\sup _{t} \int_{0}^{1}|k(t, s)| d s<\infty$.

(A3) $f \in C(I)$.

(A4) $\psi(s, x)$ is continuous in $s \in I$ and Lipschitz continuous in $x \in(-\infty, \infty)$, i.e., there exists a constant $C_{1}>0$ for which

$$
\left|\psi\left(s, x_{1}\right)-\psi\left(s, x_{2}\right)\right| \leq C_{1}\left|x_{1}-x_{2}\right| \quad \text { for all } \quad x_{1}, x_{2} \in(-\infty, \infty) .
$$

(A5) the partial derivative $\psi^{(0,1)}$ of $\psi$ with respect to the second variable exists, is uniformly bounded, and is Lipschitz continuous in $x \in(-\infty, \infty)$, i.e., there exist a constant $M>0$ such that

$$
\left|\psi^{(0,1)}(t, y)\right| \leq M, \quad(t, y) \in[0,1] \times(-\infty, \infty),
$$

and a constant $C_{2}>0$ such that

$$
\left|\psi^{(0,1)}\left(t, x_{1}\right)-\psi^{(0,1)}\left(t, x_{2}\right)\right| \leq C_{2}\left|x_{1}-x_{2}\right| \quad \text { for } \quad x_{1}, x_{2} \in(-\infty, \infty) .
$$

In the case of the nonlinear Hammerstein equation, the fast algorithm described in section 2 which is based upon the truncation strategy (2.22) must be modified, since the basis function $w_{i j}$ appears under the nonlinear term $\psi$. To circumvent this difficulty, we first transform (3.1) as follows: Defining

$$
z(t)=\psi(t, u(t))
$$

and substituting it into (3.1), we get

$$
u(t)=f(t)+\int_{0}^{1} k(t, s) z(s) d s .
$$

Equations (3.3) and (3.4) give

$$
z(t)=\psi\left(t, f(t)+\int_{0}^{1} k(t, s) z(s) d s\right) .
$$

As in the Fredholm case, we seek an approximate solution $z_{n}$ of $z$ satisfying (3.5) in the form

$$
z_{n}(t):=\sum_{(i, j) \in U_{n}} z_{i j} w_{i j}(t),
$$

and $z_{i j}$ are found by requiring that

$$
\left\langle\ell_{i^{\prime} j^{\prime}}, z_{n}(t)\right\rangle=\left\langle\ell_{i^{\prime} j^{\prime}}, \psi\left(t, f+K z_{n}(t)\right)\right\rangle, \quad\left(i^{\prime}, j^{\prime}\right) \in U_{n} .
$$

Once $z_{n}$ is found, the approximate solution $u_{n}$ of (3.1) can be found from (3.4), namely,

$$
u_{n}(t)=f(t)+\int_{0}^{1} k(t, s) z_{n}(s) d s .
$$

It should be pointed out that the substitution technique described between (3.3) and (3.5) was first introduced in [19], [17], and [18], and it was also used in conjunction 
with other methods in [12] and [15]. Let $P_{n}$ be an interpolation projection mapping $C(I)$ onto $\mathbf{X}_{n}$ which is defined by

$$
P_{n}(x)(t):=\sum_{(i, j) \in U_{n}}\left\langle\ell_{i j}, x\right\rangle w_{i j}(t), \quad x \in C(I) .
$$

Then

$$
P_{n} x \rightarrow x \quad \text { as } n \rightarrow \infty \text { and for } x \in C(I)
$$

from which we obtain by the uniform bounded principle,

$$
\sup _{n}\left\|P_{n}\right\|_{\infty}<M, \quad \text { where } M \text { is independent of } n .
$$

With

$$
T(x)(t):=f(t)+K x(t), \quad x \in L^{\infty}(I), t \in I,
$$

(3.5) can be written as

$$
z=\Psi T(z)
$$

whereas (3.6) can be written in operator form as

$$
z_{n}=P_{n} \Psi T\left(z_{n}\right), \quad z_{n} \in \mathbf{X}_{n} .
$$

Under the assumptions (A1)-(A5) along with the condition that 1 is not an eigenvalue of the linear operator $(\Psi T)^{\prime}(z)$, the Fréchet derivative of $\Psi T$ at $z$, it was shown [19] that (3.8) has an isolated solution. Similarly, one may invoke Theorem 1 of [19] to show the existence of a solution $z_{n}$ of (3.9). Alternatively, one may utilize Theorem 2 of Vainikko [22], as was done in [15], to obtain the following.

THEOREM 3.1. Let $z$ be an isolated solution of (3.8). Assume that 1 is not an eigenvalue of the linear operator $(\Psi T)^{\prime}(z)$, where $(\Psi T)^{\prime}(z)$ denotes the Fréchet derivative of $\Psi T$ at $z$. Then the wavelet collocation approximation equation (3.9) has a unique solution $z_{n}$ in the ball $B(z, \delta):=\left\{c \in C(I):\|x-z\|_{\infty} \leq \delta\right\}$ for some $\delta>0$ and for sufficiently large $n$. Moreover, there exists a constant $0<q<1$, independent of $n$, such that

$$
\frac{\alpha_{n}}{1+q} \leq\left\|z_{n}-z\right\|_{\infty} \leq \frac{\alpha_{n}}{1-q}
$$

where $\alpha_{n}:=\left\|\left(I-\left(P_{n} \Psi T\right)^{\prime}(z)\right)^{-1}\left(\left(P_{n} \Psi T\right)(z)-\Psi T(z)\right)\right\|_{\infty}$. Finally

$$
E_{n}(z) \leq\left\|z_{n}-z\right\|_{\infty} \leq C E_{n}(z),
$$

where $C$ is a constant independent of $n$ and $E_{n}(z)=\inf _{u \in \mathbf{X}_{n}}\|z-u\|_{\infty}$.

Note that for the polynomial wavelets described in section 2, and for $z \in W^{\kappa, \infty}(I)$,

$$
E_{n}(z)=\mathcal{O}\left(\frac{1}{\mu^{n \kappa}}\right) .
$$

Copyright $@$ by SIAM. Unauthorized reproduction of this article is prohibited. 
3.1. A fast wavelet collocation method. In this subsection, we describe a fast wavelet collocation method for Hammerstein equations. In order to find the solution $z_{n}$ of (3.9), one must more likely use an iterative method. Here we consider the Newton's method for an illustration. First, for each $\left(i^{\prime}, j^{\prime}\right) \in U_{n}$ and $\mathbf{z}_{n}:=\left[z_{i j}\right]_{(i, j) \in U_{n}}$ with $z_{n}=\sum_{(i, j) \in U_{n}} z_{i j} w_{i j}$, we let

$$
\begin{aligned}
F_{i^{\prime} j^{\prime}}\left(\mathbf{z}_{n}\right) & :=\left\langle\ell_{i^{\prime} j^{\prime}}, z_{n}(t)\right\rangle-\left\langle\ell_{i^{\prime} j^{\prime}}, \psi\left(t, f+K z_{n}(t)\right)\right\rangle \\
& =\left\langle\ell_{i^{\prime} j^{\prime}}, \sum_{(i, j) \in U_{n}} z_{i j} w_{i j}(t)\right\rangle-\left\langle\ell_{i^{\prime} j^{\prime}}, \psi\left(t, f+K \sum_{(i, j) \in U_{n}} z_{i j} w_{i j}(t)\right)\right\rangle
\end{aligned}
$$

and $\mathbf{F}\left(\mathbf{z}_{n}^{(k)}\right):=\left[F_{i j}\left(\mathbf{z}_{n}^{(k)}\right)\right]_{(i, j) \in U_{n}}$. Denote the Jacobian of $\mathbf{F}\left(\mathbf{z}_{n}^{(k)}\right)$ by $\mathbf{J}\left(\mathbf{z}_{n}\right)$ and its entries by $J_{i^{\prime} j^{\prime}, \alpha \beta}\left(\mathbf{z}_{n}\right)$. These entries are computed from

$$
\begin{aligned}
J_{i^{\prime} j^{\prime}, \alpha \beta}\left(\mathbf{z}_{n}\right) & :=\frac{\partial F_{i^{\prime} j^{\prime}}\left(\mathbf{z}_{n}\right)}{\partial z_{\alpha \beta}} \\
(3.12) & =\left\langle\ell_{i^{\prime} j^{\prime}}, w_{\alpha \beta}(t)\right\rangle-\left\langle\ell_{i^{\prime} j^{\prime}}, \psi^{(0,1)}\left(t, f+\sum_{(i, j) \in U_{n}} z_{i j} K w_{i j}(t)\right) \cdot K w_{\alpha \beta}(t)\right\rangle,
\end{aligned}
$$

with $\left(i^{\prime}, j^{\prime}\right),(\alpha, \beta) \in U_{n}$. The first term in the last equation is simplified by the semibiorthogonality of (2.14). The second term points to the significance of the transformed "linearized" equation (3.5). Notice that wavelets $w_{i j}$ and $w_{\alpha \beta}$ appear directly under the linear operator $K$. This enables the present method to use the block truncation strategies of $\mathrm{Chen}, \mathrm{Wu}$, and $\mathrm{Xu}$ described in the last section. More specifically, recalling (2.8)-(2.12), the second term of (3.12) can be written as

$$
\begin{aligned}
&\left\langle\ell_{i^{\prime} j^{\prime}},\right.\left.\psi^{(0,1)}\left(t, f+\sum_{(i, j) \in U_{n}} z_{i j} K w_{i j}(t)\right) \cdot K w_{\alpha \beta}(t)\right\rangle \\
&= \sum_{s \in Z_{k+1}} c_{j s} \psi^{(0,1)}\left(t_{s}, f\left(t_{s}\right)+\sum_{(i, j) \in U_{n}} z_{i j} K w_{i j}\left(t_{s}\right)\right) \cdot K w_{\alpha \beta}\left(t_{s}\right) \\
&= \sum_{s \in Z_{k+1}} c_{j s} \psi^{(0,1)}\left(t_{s}, f\left(t_{s}\right)+\sum_{(i, j) \in U_{n}} z_{i j} \int_{0}^{1} k\left(t_{s}, s\right) w_{i j}(s) d s\right) \\
& \times \int_{0}^{1} k\left(t_{s}, s\right) w_{\alpha \beta}(s) d s .
\end{aligned}
$$

We recall assumption (A5), and, for future reference, we label the uniform boundedness of $\psi^{(0,1)}(t, y)$ as

$$
\left|\psi^{(0,1)}(t, y)\right| \leq M \quad \text { for }(t, y) \in[0,1] \times(-\infty, \infty) .
$$

Lemma 3.2. Assume that the kernel $k(t, s)$ satisfies (2.19). If there is a constant $\nu>1$ such that

$$
\operatorname{dist}\left(S_{\alpha \beta}, S_{i^{\prime} j^{\prime}}\right) \geq \nu\left(d_{\alpha}+d_{i^{\prime}}\right),
$$

then there exists a positive constant $c_{1}$ such that

$$
\left|J_{i^{\prime} j^{\prime}, \alpha \beta}\left(\mathbf{z}_{n}\right)\right| \leq c_{1}\left(d_{\alpha} d_{i^{\prime}}\right)^{\kappa} \sum_{s \in S_{i^{\prime} j^{\prime}}} \int_{S_{\alpha \beta}} \frac{1}{|s-t|^{2 \kappa+\sigma}} d t,
$$

where $c_{1}=\frac{M \theta \theta_{1}^{2}}{\kappa^{\frac{2 d}{1-\nu^{-1}}}}$ in which $\theta, \theta_{1}$, and $\sigma$ are defined as in Lemma 2.1.

Copyright $@$ by SIAM. Unauthorized reproduction of this article is prohibited. 
Proof. First, note that (3.15) implies that supports $S_{\alpha \beta}$ and $S_{i^{\prime} j^{\prime}}$ are "separated from the diagonal," the concept defined in [2]. Hence, to estimate the size of $J_{i^{\prime} j^{\prime}, \alpha \beta}\left(\mathbf{z}_{n}\right)$, we consider (3.13). By virtue of (3.14) and Lemma 2.1,

$$
\begin{aligned}
\left|J_{i^{\prime} j^{\prime}, \alpha \beta}\left(\mathbf{z}_{n}\right)\right| & =\left|\left\langle\ell_{i^{\prime} j^{\prime}}, \psi^{(0,1)}\left(t, f+\sum_{(i, j) \in U_{n}} z_{i j} K w_{i j}(t)\right) \cdot K w_{\alpha \beta}(t)\right\rangle\right| \\
& \leq M\left|\left\langle\ell_{i^{\prime} j^{\prime}}, K w_{\alpha \beta}(t)\right\rangle\right| \\
& \leq M \frac{\theta \theta_{1}^{2} e^{\frac{2 d}{1-r^{-1}}}}{\kappa^{2}\left(1-r^{-} 1\right)^{\sigma}}\left(d_{\alpha} d_{i^{\prime}}\right)^{\kappa} \sum_{s \in S_{i^{\prime} j^{\prime}}} \int_{S_{\alpha \beta}} \frac{1}{|s-t|^{2 \kappa+\sigma}} d t \\
& =c_{1}\left(d_{\alpha} d_{i^{\prime}}\right)^{\kappa} \sum_{s \in S_{i^{\prime} j^{\prime}}} \int_{S_{\alpha \beta}} \frac{1}{|s-t|^{2 \kappa+\sigma}} d t .
\end{aligned}
$$

Once the estimate (3.14) is given, the block truncation strategy of Chen, Wu, and $\mathrm{Xu}$ [9], also described in section 2, can be applied to the Jacobian matrix. Namely, let $p=\left\{p_{i^{\prime} i}: i^{\prime}, i \in Z_{n+1}\right\}$ be the sequence defined in Theorem 2.2. Now, partition the Jacobian matrix as follows: $\mathbf{J}\left(\mathbf{z}_{n}\right)=\left[\mathbf{J}_{i^{\prime} \alpha}\left(\mathbf{z}_{n}\right): i^{\prime}, \alpha \in Z_{n+1}\right]$, where $\mathbf{J}_{i^{\prime} \alpha}\left(\mathbf{z}_{n}\right):=$ $\left[J_{i^{\prime} j^{\prime}, \alpha \beta}\left(\mathbf{z}_{n}\right): j^{\prime} \in Z_{f\left(i^{\prime}\right)}, \beta \in Z_{f(\alpha)}\right]$. Define $\widetilde{\mathbf{J}}\left(\mathbf{z}_{n}\right):=\left[\widetilde{\mathbf{J}}_{i^{\prime}, \alpha}\left(\mathbf{z}_{n}\right): i^{\prime}, \alpha \in Z_{n+1}\right]$, where

$$
\widetilde{\mathbf{J}}_{i^{\prime}, \alpha}\left(\mathbf{z}_{n}\right):=\left[\mathbf{J}\left(\mathbf{z}_{n}\right)(p)_{q^{\prime} q}^{i \alpha}: q^{\prime}, q \in Z_{\mu^{\min \left\{i^{\prime}, \alpha\right\}-1}}\right]
$$

and

$$
\mathbf{J}\left(\mathbf{z}_{n}\right)(p)_{q^{\prime} q}^{i \alpha}= \begin{cases}\mathbf{J}\left(\mathbf{z}_{n}\right)_{q^{\prime} q}^{i \alpha}, & \left|q^{\prime}-q\right| \leq p_{i^{\prime} i} \\ 0 & \text { otherwise }\end{cases}
$$

where $\mathbf{J}\left(\mathbf{z}_{n}\right)(p)_{q^{\prime} q}^{i \alpha}$ is defined similarly to the method in [9] which was stated in (2.21).

Lemma 3.3. Give $\delta>0$. Let parameters $p$ be defined as in Theorem 2.2. Then $n \in \mathbf{N}$ can be chosen so that, for $\mathbf{z} \in \mathbf{R}^{f(n)}$,

$$
\|\mathbf{J}(\mathbf{z})-\widetilde{\mathbf{J}}(\mathbf{z})\|_{\infty} \leq \delta .
$$

Proof. Let $\nu>1, a>0$, and $0 \leq \sigma^{\prime}<1-\sigma$. For $i^{\prime}, \alpha \in Z_{n+1}, j^{\prime} \in Z_{\omega\left(i^{\prime}\right)}$, and $\beta \in Z_{\omega(\alpha)}$ are such that $j^{\prime}=\mu\left(\mathbf{e}^{\prime}\right) r+\ell^{\prime}, \beta \in \mu\left(\mathbf{e}^{\alpha}\right) r+\ell^{\alpha}, \mathbf{e}^{\prime} \in Z_{\mu}^{i^{\prime}-1}, \mathbf{e}^{\alpha} \in Z_{\mu}^{\alpha-1}$, $\ell^{\prime}, \ell^{\alpha} \in Z_{r}$, where, recall, $r=\kappa(\mu-1)$. Define $q^{\prime}=\mu\left(\mathbf{e}^{\prime}\right)$ and $q^{\alpha}=\left\lfloor\frac{\mu\left(\mathbf{e}^{\alpha}\right)}{\mu^{\alpha-i^{\prime}}}\right\rfloor$ when $\alpha>i^{\prime}$ and $q^{\prime}=\left\lfloor\frac{\mu\left(\mathbf{e}^{\prime}\right)}{i^{i^{\prime}}-\alpha}\right\rfloor$ and $q^{\alpha}=\mu\left(\mathbf{e}^{\alpha}\right)$ when $\alpha<i^{\prime}$. Here for a constant $c,\lfloor c\rfloor$ denotes the largest integer not exceeding $c$. Also, with $\frac{k-\sigma^{\prime}}{2 \kappa-\sigma^{\prime}}<b^{\prime} \leq 1,0<\sigma^{\prime}<1-\sigma$, let $\epsilon_{i^{\prime} \alpha}=\max \left\{a \mu^{-\alpha+b^{\prime}\left(n-i^{\prime}\right)}, \nu\left(d_{i^{\prime}}+d_{\alpha}\right)\right\}$. Lemmas 3.1 and 3.2 of [9] then yield that $K_{i^{\prime} j^{\prime}, \alpha \beta} \in \mathbf{K}_{q^{\prime} q^{\alpha}}^{i^{\prime} \alpha}$ and $\operatorname{dist}\left(S_{i^{\prime} j^{\prime}}, S_{\alpha, \beta}\right) \leq \epsilon_{i^{\prime} \alpha}$. The last inequality, together with parameters $p_{i^{\prime} i}$ defined in Theorem 2.2, with $i$ replaced by $\alpha$, can be used, as was done in the proof of Theorem 3.3 in [9], to show $\left|q^{\prime}-q^{\alpha}\right| \leq p_{i^{\prime} \alpha}$, and thus with the truncation strategy $(3.17), \mathbf{J}\left(\mathbf{z}_{n}\right)(p)_{q^{\prime} q}^{i \alpha}=\mathbf{J}\left(\mathbf{z}_{n}\right)_{q^{\prime} q}^{i \alpha}$. Thus, one may invoke Lemma 3.2 of [7] to ensure that there exists a positive constant $c$ such that for all $i^{\prime}, \alpha \in Z_{n+1}$, and all $\mathbf{z} \in \mathbf{R}^{f(n)}$,

$$
\left\|\mathbf{J}_{i^{\prime} \alpha}(\mathbf{z})-\widetilde{\mathbf{J}}_{i^{\prime} \alpha}(\mathbf{z})\right\|_{\infty} \leq c \epsilon_{i^{\prime} \alpha}^{-\left(2 \kappa-\sigma^{\prime}\right)} \mu^{-k\left(i^{\prime}+\alpha\right)} .
$$

Once estimate (3.18) is obtained, then arguing in the same way as in the proof of Lemma 4.2 (equation (4.11) of [7]),

$$
\|(\mathbf{J}(\mathbf{z})-\widetilde{\mathbf{J}}(\mathbf{z})) \mathbf{v}\|_{\infty} \leq c_{1} \mu^{-\sigma^{\prime} n}\|\mathbf{v}\|_{\infty}
$$

Copyright (c) by SIAM. Unauthorized reproduction of this article is prohibited. 
where $v \in L^{\infty}(I), v:=\sum_{(i, j) \in U_{n}} v_{i j} w_{i j}, \mathbf{v}:=\left[v_{i j}:(i, j) \in U_{n}\right]^{T} \in \mathbf{R}^{f(n)}$, and $c_{1}$ is a constant independent of $n$. Hence, this lemma is proved by selecting $n$ so that $\left|c_{1} \mu^{-\sigma^{\prime} n}\right|<\delta$.

LEMma 3.4. Let the compressed Jacobian $\widetilde{\mathbf{J}}\left(\mathbf{z}_{n}\right)$ be constructed as in (3.17) with the parameters $p_{i^{\prime} i}$ defined as in Theorem 2.2. If $N\left(\widetilde{\mathbf{J}}\left(\mathbf{z}_{n}\right)\right)$ denotes the number of nonzero elements in $\widetilde{\mathbf{J}}\left(\mathbf{z}_{n}\right)$, then

$$
N\left(\widetilde{\mathbf{J}}\left(\mathbf{z}_{n}\right)\right)=\mathcal{O}\left(f(n) \log ^{\tau} f(n)\right),
$$

where $\tau=1$, except for $b^{\prime}=1$, in which case $\tau=2$. Here $\frac{k-\sigma^{\prime}}{2 \kappa-\sigma^{\prime}}<b^{\prime} \leq 1,0<\sigma^{\prime}<$ $1-\sigma$.

Proof. This follows immediately from Theorem 4.6 of [7].

The Newton's method finds the solution $z_{n}$ of (3.6) or, equivalently, (3.9) as follows: Starting with an initial vector $\mathbf{z}_{n}^{(0)}=\left[z_{i j}^{0}\right]$, which we assume to be sufficiently close to the solution $\mathbf{z}_{n}=\left[z_{i j}\right]$ of (3.6), one finds $\mathbf{z}_{n}, n \geq 1$, the solution of $\mathbf{F}\left(\mathbf{z}_{n}\right)=\mathbf{0}$ by the iteration

$$
\mathbf{z}_{n}^{(k+1)}=\mathbf{z}_{n}^{(k)}-\mathbf{J}^{-1}\left(\mathbf{z}_{n}^{(k)}\right) \mathbf{F}\left(\mathbf{z}_{n}^{(k)}\right) .
$$

Of course, one does not invert the Jacobian $\mathbf{J}\left(\mathbf{z}_{n}^{(k)}\right)$ directly for each $n$, but rather solves for $\mathbf{y}_{n}^{(k)}$ in

$$
\mathbf{J}\left(\mathbf{z}_{n}^{(k)}\right) \mathbf{y}_{n}^{(k)}=\mathbf{F}\left(\mathbf{z}_{n}^{(k)}\right)
$$

and (3.16) is implemented as

$$
\mathbf{z}_{n}^{(k+1)}=\mathbf{z}_{n}^{(k)}-\mathbf{y}_{n}^{(k)} .
$$

Now, we are ready to propose a fast wavelet collocation method for the Hammerstein equation. Let (3.17) and (3.18) be replaced by

$$
\widetilde{\mathbf{J}}\left(\tilde{\mathbf{z}}_{n}^{(k)}\right) \tilde{\mathbf{y}}_{n}^{(k)}=\mathbf{F}\left(\tilde{\mathbf{z}}_{n}^{(k)}\right)
$$

and

$$
\tilde{\mathbf{z}}_{n}^{(k+1)}=\tilde{\mathbf{z}}_{n}^{(k)}-\tilde{\mathbf{y}}_{n}^{(k)},
$$

respectively, where $\tilde{\mathbf{z}}_{n}^{(0)}=\mathbf{z}_{n}^{(0)}$. We call $\tilde{\mathbf{z}}_{n}^{(k)}$ the kth iterate of the fast collocation method arising from the truncation strategy (3.17). The following lemma plays a critical role in the accuracy of the fast collocation method. Here, in addition to assumptions (A1)-(A5), we assume the following:

(A6) The Hessian $\mathbf{H}(\mathbf{a})$ of $\mathbf{F}$ is uniformly bounded for all $\mathbf{a} \in \mathbf{R}^{f(n)}$.

Lemma 3.5. Let $\delta>0$, and let $p$ be the parameter defined in Theorem 2.2. Then there exists $n \in \mathbf{N}, \mathbf{z}_{n}^{(0)} \in \mathbf{R}^{f(n)}$ so that, for all $k \in \mathbf{N}$, we have

$$
\left\|\tilde{\mathbf{z}}_{n}^{(k)}-\mathbf{z}_{n}^{(k)}\right\|_{\infty}<\delta .
$$

Proof. We prove this by induction. With $k=1$, since $\tilde{\mathbf{z}}_{n}^{(0)}=\mathbf{z}_{n}^{(0)}$,

$$
\begin{aligned}
\tilde{\mathbf{z}}_{n}^{(1)}-\mathbf{z}_{n}^{(1)} & =\tilde{\mathbf{y}}_{n}^{(1)}-\mathbf{y}_{n}^{(1)} \\
& =\left[\widetilde{\mathbf{J}}\left(\mathbf{z}_{n}^{(0)}\right)^{-1}-\mathbf{J}\left(\mathbf{z}_{n}^{(0)}\right)^{-1}\right] \mathbf{F}\left(\mathbf{z}_{n}^{(0)}\right) \\
& =\widetilde{\mathbf{J}}\left(\mathbf{z}_{n}^{(0)}\right)^{-1}\left[\mathbf{J}\left(\mathbf{z}_{n}^{(0)}\right)-\widetilde{\mathbf{J}}\left(\mathbf{z}_{n}^{(0)}\right)\right] \mathbf{J}\left(\mathbf{z}_{n}^{(0)}\right)^{-1} \mathbf{F}\left(\mathbf{z}_{n}^{(0)}\right) .
\end{aligned}
$$

Copyright (C) by SIAM. Unauthorized reproduction of this article is prohibited. 
Taking the norm on both sides and choosing $n \in \mathbf{N}$ by Lemma 3.3, so that $\| \mathbf{J}\left(\mathbf{z}_{n}^{(0)}\right)-$ $\widetilde{\mathbf{J}}\left(\mathbf{z}_{n}^{(0)}\right) \|_{\infty} \leq \delta /\left\{\left\|\widetilde{\mathbf{J}}\left(\mathbf{z}_{n}^{(0)}\right)^{-1}\right\|_{\infty}\left\|\mathbf{J}\left(\mathbf{z}_{n}^{(0)}\right)^{-1}\right\|_{\infty}\left\|\mathbf{F}\left(\mathbf{z}_{n}^{(0)}\right)\right\|_{\infty}\right\}$, we get $\left\|\tilde{\mathbf{z}}_{n}^{(1)}-\mathbf{z}_{n}^{(1)}\right\|_{\infty}<\delta$. Now assume that (3.21) is true for $k-1$. Then

$$
\begin{aligned}
& \tilde{\mathbf{z}}_{n}^{(k)}-\mathbf{z}_{n}^{(k)}=\tilde{\mathbf{z}}_{n}^{(k-1)}-\mathbf{z}_{n}^{(k-1)}-\left(\tilde{\mathbf{y}}_{n}^{(k-1)}-\mathbf{y}_{n}^{(k-1)}\right) \\
&=\tilde{\mathbf{z}}_{n}^{(k-1)}-\mathbf{z}_{n}^{(k-1)}+\left[\mathbf{J}^{-1}\left(\tilde{\mathbf{z}}_{n}^{(k-1)}\right)-\widetilde{\mathbf{J}}^{-1}\left(\tilde{\mathbf{z}}_{n}^{(k-1)}\right)\right] \mathbf{F}\left(\tilde{\mathbf{z}}_{n}^{(k-1)}\right) \\
&\left.-\mathbf{J}^{-1}\left(\tilde{\mathbf{z}}_{n}^{(k-1)}\right)\left[\mathbf{F}\left(\tilde{z}_{n}^{(k-1)}\right)-\mathbf{F}\left(\mathbf{z}_{n}^{(k-1)}\right)\right]-\left[\mathbf{J}^{-1}\left(\tilde{z}_{n}^{(k-1)}\right)-\mathbf{J}^{-1}\left(\mathbf{z}_{n}^{(k-1)}\right)\right] \mathbf{F}\left(\mathbf{z}_{n}^{(k-1)}\right)\right] \\
& \quad:=I+I I+I I I+I V .
\end{aligned}
$$

For $I$, by induction, let $n_{1} \in \mathbf{N}$ be such that $\left\|\tilde{\mathbf{z}}_{n_{1}}^{(k-1)}-\mathbf{z}_{n_{1}}^{(k-1)}\right\|_{\infty}<\delta / 4$. For $I I$, $(3.25)$

$$
\left\|\left[\mathbf{J}^{-1}\left(\tilde{\mathbf{z}}_{n}^{(k-1)}\right)-\widetilde{\mathbf{J}}^{-1}\left(\tilde{\mathbf{z}}_{n}^{(k-1)}\right)\right] \mathbf{F}\left(\tilde{\mathbf{z}}_{n}^{(k-1)}\right)\right\|_{\infty} \leq c_{1}\left\|\widetilde{\mathbf{J}}\left(\tilde{\mathbf{z}}_{n}^{(k-1)}\right)-\mathbf{J}\left(\tilde{\mathbf{z}}_{n}^{(k-1)}\right)\right\|_{\infty},
$$

where $c_{1}=\left\|\mathbf{J}^{-1}\left(\tilde{\mathbf{z}}_{n}^{(k-1)}\right)\right\|_{\infty}\left\|\widetilde{\mathbf{J}}^{-1}\left(\tilde{\mathbf{z}}_{n}^{(k-1)}\right)\right\|_{\infty}\left\|\mathbf{F}\left(\tilde{\mathbf{z}}_{n}^{(k-1)}\right)\right\|_{\infty}$. By Lemma 3.3, choose $n_{2} \in \mathbf{N}$, so that the right side of (3.22) with $n=n_{2}$ can be made $<\frac{\delta}{4}$. For $I I I$,

$$
\left\|\mathbf{J}^{-1}\left(\tilde{\mathbf{z}}_{n}^{(k-1)}\right)\left[\mathbf{F}\left(\tilde{z}_{n}^{(k-1)}\right)-\mathbf{F}\left(\mathbf{z}_{n}^{(k-1)}\right)\right]\right\|_{\infty} \leq c_{2}\left\|\tilde{\mathbf{z}}_{n}^{(k-1)}-\mathbf{z}_{n}^{(k-1)}\right\|_{\infty},
$$

where $c_{2}=\left\|\mathbf{J}^{-1}\left(\tilde{\mathbf{z}}_{n}^{(k-1)}\right)\right\|_{\infty}\left\|\mathbf{J}\left(\tilde{\mathbf{z}}_{n}^{(k-1)}+\theta\left(\tilde{\mathbf{z}}_{n}^{(k-1)}-\mathbf{z}_{n}^{(k-1)}\right)\right)\right\|_{\infty}$ with $0<\theta<1$. Applying induction, choose $n_{3} \in \mathbf{N}$ so that the right side of (3.23) is made $<\frac{\delta}{4}$. Finally for $I V$,

$$
\begin{aligned}
& \left\|\left[\mathbf{J}^{-1}\left(\tilde{z}_{n}^{(k-1)}\right)-\mathbf{J}^{-1}\left(\mathbf{z}_{n}^{(k-1)}\right)\right] \mathbf{F}\left(\mathbf{z}_{n}^{(k-1)}\right)\right\|_{\infty} \\
& \quad \leq\left\|\mathbf{J}^{-1}\left(\tilde{z}_{n}^{(k-1)}\right)\right\|_{\infty}\left\|\mathbf{J}\left(\mathbf{z}_{n}^{(k-1)}\right)-\mathbf{J}\left(\tilde{z}_{n}^{(k-1)}\right)\right\|_{\infty}\left\|\mathbf{J}^{-1}\left(\mathbf{z}_{n}^{(k-1)}\right)\right\|_{\infty}\left\|\mathbf{F}\left(\mathbf{z}_{n}^{(k-1)}\right)\right\|_{\infty} \\
& \quad<c_{3}\left\|\tilde{\mathbf{z}}_{n}^{(k-1)}-\mathbf{z}_{n}^{(k-1)}\right\|_{\infty},
\end{aligned}
$$

where $c_{3}=\left\|\mathbf{F}\left(\mathbf{z}_{n}^{(k-1)}\right)\right\|_{\infty}\left\|\mathbf{H}\left(\tilde{\mathbf{z}}_{n}^{(k-1)}+\beta\left(\tilde{\mathbf{z}}_{n}^{(k-1)}-\mathbf{z}_{n}^{(k-1)}\right)\right)\right\|_{\infty}$ with $0<\beta<1$. By another application of the induction hypothesis and considering (3.22)-(3.24), we complete the proof of this lemma.

The following theorem establishes the convergence of the fast wavelet collocation method for the Hammerstein equation described in (3.19) and (3.20).

TheOrem 3.6. Assume that $\mathbf{z}_{n}=\left[z_{i j}\right]$ is a solution of (3.9), i.e., $\mathbf{z}_{n}=\mathbf{F}\left(\mathbf{z}_{n}\right)$. Also let $\mathbf{z}_{n}^{(0)} \in \mathbf{R}^{\omega(n)}$ be such that the Newton iteration given in (3.20)-(3.21) generates a sequence $\mathbf{z}_{n}^{(k)}$ that converges to $\mathbf{z}_{n}$ as $k \rightarrow \infty$. Then the parameter $p$ in the block truncation strategy can be chosen so that the fast wavelet collocation method described in (3.22)-(3.23) generates a sequence $\tilde{\mathbf{z}}_{n}^{(k)}$ which converges to $\mathbf{z}_{n}$ as $k \rightarrow \infty$.

Proof. This follows by noting that

$$
\tilde{\mathbf{z}}_{n}^{(k)}-\mathbf{z}_{n}=\tilde{\mathbf{z}}_{n}^{(k)}-\mathbf{z}_{n}^{(k)}+\mathbf{z}_{n}^{(k)}-\mathbf{z}_{n}
$$

and applying Lemma 3.4 to the first two terms and the convergence assumption to the remaining two terms.

With $\tilde{z}_{n}=\sum_{(i, j) \in U_{n}}\left(\tilde{\mathbf{z}}_{n}^{k}\right)_{i, j} w_{i, j}$, Theorem 3.6 guarantees that the convergence rate of the fast wavelet collocation solution $\tilde{z}_{n}$ to $z$ is the same as the rate of convergence of $z_{n}$ to $z$, which is described in Theorem 3.1. To see this, we recall that Proposition 5.6 of [10] implies

$$
\theta_{2}\left\|\tilde{\mathbf{z}}_{n}^{k}-\mathbf{z}\right\|_{\infty} \leq\left\|\tilde{z}_{n}^{k}-z\right\| \leq \theta_{3}(n+1)\left\|\mathbf{E}_{n}\left(\tilde{\mathbf{z}}_{n}^{k}-\mathbf{z}\right)\right\|_{\infty}
$$

where $\mathbf{E}_{n}=\left[\left\langle\ell_{i^{\prime} j^{\prime}}, w_{i j}\right\rangle\right]_{f(n) \times f(n)}$ and thus

$$
\left\|\tilde{z}_{n}-z\right\|_{\infty}=\mathcal{O}\left(\frac{1}{\mu^{n \kappa}}\right) .
$$

Copyright $\odot$ by SIAM. Unauthorized reproduction of this article is prohibited. 
3.2. Smooth kernel. The property (2.19) played a critical role in establishing the truncation parameters for the compressed fast wavelet collocation method. In this subsection, we briefly comment on how the same truncation parameters can be applied to the Hammerstein equations with smooth kernels. We note that the paper [1] also discusses the sparsity of the resulting linear system when a class of wavelet bases is applied to the Fredholm equation with smooth kernel. Define $G: W^{k, \infty}(I) \rightarrow$ $W^{k, \infty}(I)$ to be

$$
(G x)(t):=\int_{I} g(t, s) x(s) d s,
$$

where $g \in C^{\kappa}(I \times I)$. Let $G_{i^{\prime} j^{\prime}, i j}:=\left\langle\ell_{i^{\prime} j^{\prime}}, G w_{i j}\right\rangle$. If $\bar{t}_{i j}$ denotes the midpoint of $S_{i j}$, then expanding $g$ in the Taylor series at the midpoints of $S_{i^{\prime} j^{\prime}}$ and $S_{i j}$, and using the vanishing moment property (2.13), we obtain

$$
\begin{aligned}
\left|G_{i^{\prime} j^{\prime}, i j}\right| & =\left|\left\langle\ell_{i^{\prime} j^{\prime}}, \int_{S_{i j}} g(t, s) w_{i j}(s) d s\right\rangle\right| \\
& \leq\left|\left\langle\ell_{i^{\prime} j^{\prime}}, \int_{S_{i j}} D_{t}^{\kappa} D_{s}^{\kappa} g\left(c_{i^{\prime} j^{\prime}}, c_{i j}\right)\left(t-\bar{t}_{i j}\right)^{\kappa}\left(s-\bar{t}_{i^{\prime} j^{\prime}}\right)^{\kappa} w_{i j}(s) d s\right\rangle\right| \\
& \leq M \theta\left(\frac{d_{i^{\prime}}}{2}\right)^{\kappa}\left(\frac{d_{i}}{2}\right)^{\kappa} \int_{S_{i j}}\left|w_{i j}(s)\right| d s \\
& \leq M \theta 2^{-2 \kappa} d_{i^{\prime}}^{\kappa} d_{i}^{\kappa+\frac{1}{2}},
\end{aligned}
$$

where $c_{i^{\prime} j^{\prime}} \in S_{i^{\prime} j^{\prime}}, c_{i j} \in S_{i j}$ and $\max _{\sigma_{1}, \sigma_{2} \in I}\left|D_{t}^{\kappa} D_{s}^{\kappa} g\left(\sigma_{1}, \sigma_{2}\right)\right|<M,\left\|\ell_{i j}\right\|<\theta$.

The estimate (3.28) is similar to the estimate given in Lemma 2.1 (Lemma 3.1 in [7]) for integrals with weakly singular kernel and is independent of the distance between two supports $S_{i^{\prime} j^{\prime}}$ and $S_{i j}$. However, the truncation parameter $\epsilon$ should still be subject to the distance in order for the resulting truncated system of linear equations to be sparse. For this, see Lemma 2.4 of [1] and Theorem 4.6 of [7]. To demonstrate that the same truncation parameter used for weakly singular kernel can be used for integrals with smooth kernel, we let

$$
\mathbf{G}_{n}=\left[\mathbf{G}_{i^{\prime} i}\right]_{i^{\prime}, i \in Z_{n+1}},
$$

where

$$
\mathbf{G}_{i^{\prime} i}=\left[G_{i^{\prime} j^{\prime}, i j}\right]_{j^{\prime} \in Z_{\omega\left(i^{\prime}\right)}, j \in Z_{\omega(i)}} .
$$

Following [7], for $\epsilon>0$, define

$$
\mathbf{G}(\epsilon)_{i^{\prime} i}=\left[G(\epsilon)_{i^{\prime} j^{\prime}, i j}\right]_{j^{\prime} \in Z_{\omega\left(i^{\prime}\right)}, j \in Z_{\omega(i)}}
$$

where

$$
G(\epsilon)_{i^{\prime} j^{\prime}, i j}= \begin{cases}G_{i^{\prime} j^{\prime}, i j}, & \operatorname{dist}\left(S_{i^{\prime} j^{\prime}}, S_{i j}\right) \leq \epsilon \\ 0 & \text { otherwise. }\end{cases}
$$

The estimate in (3.28) then yields the following lemma. It is written in a form equivalent to Lemma 3.2 of [7] so that the analysis of Lemma 4.2 of [7] can be used to show a bound for $\|\mathbf{G}-\mathbf{G}(\epsilon)\|_{\infty}$, which is similar to the one given in Lemma 3.3. 
Lemma 3.7. Let $\nu>1,0<\sigma<1$, and $0 \leq \sigma^{\prime}<\min \{2 \kappa, 1-\sigma\}$. Then there exists a positive constant $c$ such that whenever $\epsilon \geq \nu\left(d_{i}+d_{i^{\prime}}\right)$,

$$
\left\|\mathbf{G}_{i^{\prime} i}-\mathbf{G}(\epsilon)_{i^{\prime} i}\right\|_{\infty} \leq c \epsilon^{-\eta}\left(d_{i^{\prime}} d_{i}\right)^{\kappa}, \quad i^{\prime}, i \in Z_{n+1},
$$

where $\eta=2 \kappa-\sigma^{\prime}$.

Proof. First,

$$
\left\|\mathbf{G}_{i^{\prime} i}-\mathbf{G}(\epsilon)_{i^{\prime} i}\right\|_{\infty}=\max _{j^{\prime} \in Z_{\omega\left(i^{\prime}\right)}} \sum_{j \in Z_{i^{\prime} j^{\prime}}(\epsilon)}\left|G_{i^{\prime} j^{\prime}, i j}\right|
$$

where

$$
Z_{i^{\prime} j^{\prime}}(\epsilon):=\left\{j: j \in Z_{\omega(i)}, \operatorname{dist}\left(S_{i j}, S_{i^{\prime} j^{\prime}}\right)>\epsilon\right\}
$$

Using (3.28),

$$
\begin{aligned}
\left\|\mathbf{G}_{i^{\prime} i}-\mathbf{G}(\epsilon)_{i^{\prime} i}\right\|_{\infty} & \leq \max _{j^{\prime} \in Z_{\omega\left(i^{\prime}\right)}} \sum_{j \in Z_{i^{\prime} j^{\prime}}(\epsilon)} M \theta 2^{-2 \kappa}\left[d\left(S_{i^{\prime} j^{\prime}}, S_{i j}\right)\right]^{\eta} \epsilon^{-\eta} d_{i^{\prime}}^{\kappa} d_{i}^{\kappa+\frac{1}{2}} \\
& \leq \max _{j^{\prime} \in Z_{\omega\left(i^{\prime}\right)}} M \theta 2^{-2 \kappa}\left[d\left(S_{i^{\prime} j^{\prime}}, S_{i j}\right)\right]^{\eta} M_{2} i(\log \mu)\left(\frac{1}{\mu^{i}}\right)^{1 / 2} \epsilon^{-\eta} d_{i^{\prime}}^{\kappa} d_{i}^{\kappa} \\
& \leq \max _{j^{\prime} \in Z_{\omega\left(i^{\prime}\right)}} M \theta 2^{-2 \kappa}\left[d\left(S_{i^{\prime} j^{\prime}}, S_{i j}\right)\right]^{\eta} M_{2}(\log \mu) \epsilon^{-\eta} d_{i^{\prime}}^{\kappa} d_{i}^{\kappa} \\
& =c \epsilon^{-\eta} d_{i^{\prime}}^{\kappa} d_{i}^{\kappa},
\end{aligned}
$$

where $c=\max _{j^{\prime} \in Z_{\omega\left(i^{\prime}\right)}} M \theta 2^{-2 \kappa}\left[d\left(S_{i^{\prime} j^{\prime}}, S_{i j}\right)\right]^{\eta} M_{2}(\log \mu)$ and we used the fact that the cardinality of $Z_{i^{\prime} j^{\prime}}(\epsilon)$ is of order $\mathcal{O}(i \log \mu)$, i.e., $\left|Z_{i^{\prime} j^{\prime}}(\epsilon)\right|=M_{2} i \log \mu$ for some $M_{2}>0$. The latter follows by observing that, with the condition $\epsilon \geq \nu\left(d_{i}+d_{i^{\prime}}\right)$, sets $S_{i j}$ which satisfy $\operatorname{dist}\left(S_{i j}, S_{i^{\prime} j^{\prime}}\right)>\epsilon$ are "separated from the diagonal," the notion defined in [1], and there are $\mathcal{O}\left(\mu^{i} \log u^{i}\right)$ many such sets.

With Lemma 3.7, we may set the truncation parameter $\epsilon_{i^{\prime} i}^{n}$ to satisfy

$$
\epsilon_{i^{\prime} i}^{n} \geq \max \left\{a \mu^{-n+b(n-i)+b^{\prime}\left(n-i^{\prime}\right)}, \nu\left(d_{i}+d_{i}^{\prime}\right)\right\},
$$

where $b, b^{\prime}$ are real numbers, $a>1$, and $\nu>1$. Then arguing exactly as in Lemma 4.2 of [7], we obtain, for $v \in W^{k, \infty}(I), \mathbf{v}=\left[v_{i j}\right]$ with $P_{n} v=\sum_{(i, j) \in U_{n}} v_{i j} w_{i j}$,

$$
\left\|\mathbf{G}_{n}-\mathbf{G}_{n}(\epsilon)\right\|_{\infty}=\mathcal{O}\left(\mu^{-\left(k+\sigma^{\prime}\right) n}\right) .
$$

Equation (3.31) is equivalent to Lemma 3.3.

3.3. Numerical results. Next, numerical results of the wavelet collocation scheme using the piecewise linear wavelet basis from section 2.2 are shown. Comparisons between the full wavelet collocation solutions and the fast wavelet collocation solutions are presented in Tables 1 and 2 using two different examples. Here, the word "full" means solving the corresponding system without the truncation strategy. For the fast wavelet collocation method, compression parameters are set with $a=0.25, \nu=1.01$, and $b^{\prime}=0.8$. Recall that these parameters are defined in Theorem 2.2 (Theorem 3.8 of [9]). We use the tilde symbol $\sim$ to represent the fast wavelet collocation results. The nonlinear system (3.9) is solved by the Newton's method first, followed by the quasi-Newton method for comparison. Programs are run on a PC with 
2.0GHz CPU and 4GB memory. We note that it is possible to relax the condition of uniform boundedness of $\psi^{(0,1)}$ assumed in (3.14) provided that an intelligent estimate of the bound can be found. Example 3.8 demonstrates this point. A careful analysis of this estimate will appear in a future paper.

Example 3.8. Consider the equation

$$
u(t)-\int_{0}^{1} e^{s+t} u^{2}(s) d s=f(t), \quad t \in[0,1]
$$

where $f(t)$ is chosen so that the isolated solution $u(t)=\cos (t)$. See the numerical results in Tables 1 and 3 .

Example 3.9. Consider the equation

$$
u(t)-\int_{0}^{1} \frac{\sin (u(s))}{\sqrt{|s-t|}} d s=f(t), \quad t \in[0,1]
$$

where $f(t)$ is chosen so that the isolated solution $u(t)=\arcsin \left(t^{3}\right)$. See the numerical results in Tables 2 and 4 .

TABLE 1

Computational results for the Hammerstein equation with smooth kernel.

\begin{tabular}{|c|c|c|c|c|c|c|c|c|}
\hline \multirow{2}{*}{$f(n)$} & \multicolumn{3}{|c|}{ Full collocation solution } & \multicolumn{4}{c|}{ Fast collocation solution } \\
\cline { 2 - 8 } & $e_{h}$ & $R_{h}$ & $T_{N}$ & $T_{Q}$ & $\widetilde{e}_{h}$ & $\widetilde{R}_{h}$ & $\widetilde{T}_{N}$ & $\widetilde{T}_{Q}$ \\
\hline 2 & $2.7795 \mathrm{e}-2$ & & 0.00 & 0.00 & $2.7795 \mathrm{e}-2$ & & 0.00 & 0.00 \\
4 & $5.7798 \mathrm{e}-3$ & 2.27 & 0.01 & 0.01 & $5.7798 \mathrm{e}-3$ & 2.27 & 0.01 & 0.01 \\
8 & $1.3863 \mathrm{e}-3$ & 2.06 & 0.03 & 0.03 & $1.3863 \mathrm{e}-3$ & 2.06 & 0.03 & 0.03 \\
16 & $3.4311 \mathrm{e}-4$ & 2.01 & 0.11 & 0.08 & $3.4311 \mathrm{e}-4$ & 2.01 & 0.10 & 0.08 \\
32 & $8.5564 \mathrm{e}-5$ & 2.00 & 0.41 & 0.26 & $8.5563 \mathrm{e}-5$ & 2.00 & 0.39 & 0.25 \\
64 & $2.1378 \mathrm{e}-5$ & 2.00 & 1.64 & 0.98 & $2.1376 \mathrm{e}-5$ & 2.00 & 1.42 & 0.08 \\
128 & $5.3435 \mathrm{e}-6$ & 2.00 & 6.51 & 3.83 & $5.3414 \mathrm{e}-6$ & 2.00 & 5.18 & 2.50 \\
256 & $1.3358 \mathrm{e}-6$ & 2.00 & 26.32 & 15.45 & $1.3336 \mathrm{e}-6$ & 2.00 & 19.20 & 8.08 \\
512 & $3.3393 \mathrm{e}-7$ & 2.00 & 104.89 & 62.20 & $3.3168 \mathrm{e}-7$ & 2.01 & 73.03 & 29.84 \\
\hline
\end{tabular}

TABLE 2

Computational results for the Hammerstein equation with weakly singular kernel.

\begin{tabular}{|c|c|c|c|c|c|c|c|c|}
\hline \multirow{2}{*}{$f(n)$} & \multicolumn{3}{|c|}{ Full collocation solution } & \multicolumn{3}{|c|}{ Fast collocation solution } \\
\cline { 2 - 9 } & $e_{h}$ & $R_{h}$ & $T_{N}$ & $T_{Q}$ & $\widetilde{e}_{h}$ & $\widetilde{R}_{h}$ & $\widetilde{T}_{N}$ & $\widetilde{T}_{Q}$ \\
\hline 2 & $1.7064 \mathrm{e}-1$ & & 0.00 & 0.00 & $1.7064 \mathrm{e}-1$ & & 0.00 & 0.00 \\
4 & $1.2076 \mathrm{e}-1$ & 0.50 & 0.00 & 0.00 & $1.2076 \mathrm{e}-1$ & 0.50 & 0.00 & 0.00 \\
8 & $3.2859 \mathrm{e}-2$ & 1.88 & 0.02 & 0.01 & $3.2859 \mathrm{e}-2$ & 1.88 & 0.02 & 0.01 \\
16 & $9.9151 \mathrm{e}-3$ & 1.73 & 0.06 & 0.04 & $9.9151 \mathrm{e}-3$ & 1.73 & 0.05 & 0.03 \\
32 & $2.8965 \mathrm{e}-3$ & 1.77 & 0.22 & 0.13 & $2.8964 \mathrm{e}-3$ & 1.77 & 0.21 & 0.12 \\
64 & $7.8456 \mathrm{e}-4$ & 1.88 & 0.86 & 0.48 & $7.8443 \mathrm{e}-4$ & 1.88 & 0.78 & 0.42 \\
128 & $2.0275 \mathrm{e}-4$ & 1.95 & 3.78 & 1.81 & $2.0264 \mathrm{e}-4$ & 1.95 & 3.39 & 1.53 \\
256 & $5.2075 \mathrm{e}-5$ & 1.96 & 15.63 & 8.43 & $5.1961 \mathrm{e}-5$ & 1.96 & 13.60 & 6.95 \\
512 & $1.3238 \mathrm{e}-5$ & 1.98 & 61.75 & 33.36 & $1.3137 \mathrm{e}-5$ & 1.98 & 53.06 & 24.11 \\
\hline
\end{tabular}

Denote a numerical error by $e_{h}=\left\|u-u_{n}\right\|_{\infty}$ and an order of convergence by $R_{h}=\log _{2}\left(\frac{e_{h}}{e_{h / 2}}\right)$.

Note that for piecewise linear wavelets, $R_{h}=2$ (see, e.g., [8]). This theoretical order of convergence is attained in Examples 3.8 and 3.9 by both the full collocation method and the fast wavelet collocation method. Let $T_{N}$ and $T_{Q}$ be the computing time measured in seconds by using the Newton's method and the quasi-Newton 
method, respectively. A comparison between the iteration numbers for the two methods is listed in Tables 3 and 4 for Examples 3.8 and 3.9, respectively, where $I_{N}$ and $I_{Q}$ are the iteration numbers for the Newton's method and the quasi-Newton method, respectively.

TABLE 3

A comparison of iteration numbers of the numerical schemes for smooth kernel.

\begin{tabular}{c|ccccccccc}
\hline$f(n)$ & 2 & 4 & 8 & 16 & 32 & 64 & 128 & 256 & 512 \\
\hline$I_{N}$ or $\widetilde{I}_{N}$ & 5 & 5 & 5 & 5 & 5 & 5 & 5 & 5 & 5 \\
\hline$I_{Q}$ or $\widetilde{I}_{Q}$ & 21 & 22 & 22 & 22 & 22 & 22 & 22 & 22 & 22 \\
\hline
\end{tabular}

TABLE 4

A comparison of iteration numbers of the numerical schemes for weakly singular kernel.

\begin{tabular}{c|ccccccccc}
\hline$f(n)$ & 2 & 4 & 8 & 16 & 32 & 64 & 128 & 256 & 512 \\
\hline$I_{N}$ or $\widetilde{I}_{N}$ & 6 & 6 & 6 & 7 & 7 & 7 & 8 & 8 & 8 \\
\hline$I_{Q}$ or $\widetilde{I}_{Q}$ & 12 & 14 & 15 & 20 & 30 & 38 & 41 & 43 & 43 \\
\hline
\end{tabular}

TABLE 5

Number of nonzeros in the compressed Jacobian matrix associated with Examples 3.8 and 3.9 .

\begin{tabular}{c|ccccc}
\hline$f(n)$ & 32 & 64 & 128 & 256 & 512 \\
\hline Number of nonzeros & 928 & 2832 & 7872 & 20464 & 50720 \\
\hline Ratio of nonzeros (in \%) & 90.625 & 69.141 & 48.048 & 31.226 & 19.348 \\
\hline
\end{tabular}

We see that the computing time of the fast wavelet collocation method is less than the computing time of the full collocation method. Furthermore, we observe that although the number of iterations using the quasi-Newton method are more than the required number of iterations of the Newton's method, the computing times of the quasi-Newton method are faster in the two examples tested.

Figure 7 represents the structures of the Jacobian matrices with varied values of $f(n)$ which are constructed using the block truncation strategy. The corresponding structures for Example 3.8 are similar. Sparsity occurs when one solves the Hammerstein equation with smooth or weakly singular kernel. Table 5 shows the number of nonzeros observed in the compressed Jacobian matrices. These numbers are consistent with the theoretical estimate given in Lemma 3.4.

4. Multilevel augmentation method. A first form of a multilevel augmentation method appeared in the paper [5] in connection with the Fredholm integral equation of the second kind. A multilevel augmentation method is used with a basis having a multiresolution structure such as wavelet functions. Each multilevel augmentation method solves an underlying equation at a low dimensional subspace and enhances the accuracy of its approximation by adding successively to the solution correction terms which can be obtained by solving systems at a low dimension. The method was recently extended to obtain a fast algorithm for a class of nonlinear Hammerstein equations [8]. In this section, we present another multilevel augmentation method for Hammerstein equations which is based upon the "linearization" technique explored in section 3. The block truncation strategy is also used to construct the Jacobian matrices. Numerical examples are provided to demonstrate the convergence of the new multilevel augmentation method and the effectiveness of the truncation strategy. 


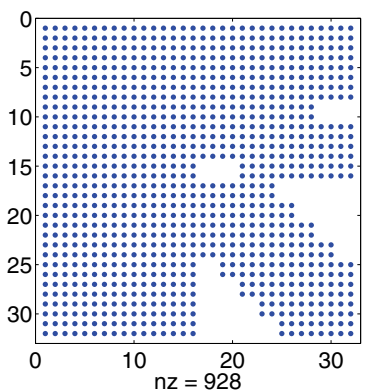

(a) $f(n)=32$

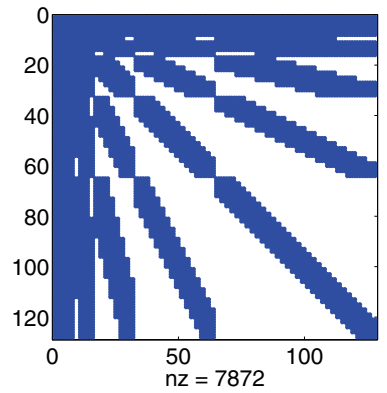

(c) $f(n)=128$

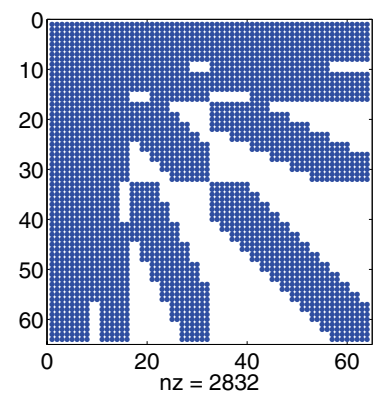

(b) $f(n)=64$

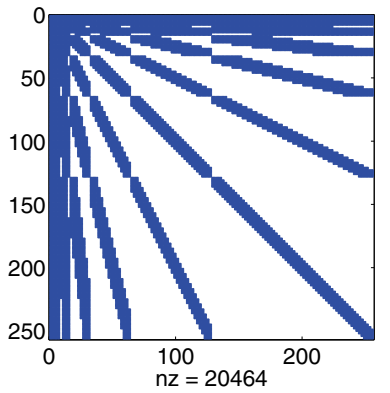

(d) $f(n)=256$

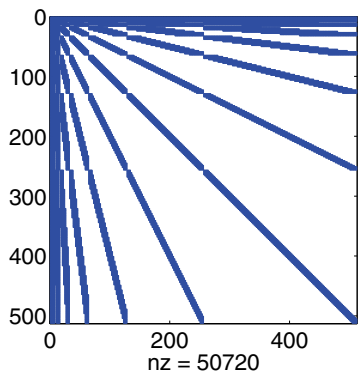

(e) $f(n)=512$

Fig. 7. Sparsity of corresponding Jacobian matrix of linearized Hammerstein equations associated with Examples 3.8 and 3.9 .

Recall the decomposition of the subspace $\mathbf{X}_{n}$ for $L^{\infty}(E)$, i.e., with $n=k+m$,

$$
\mathbf{X}_{k+m}=\mathbf{X}_{k} \oplus \mathbf{W}_{k+1} \oplus \cdots \oplus \mathbf{W}_{k+m} .
$$

Our method goes as follows. First, we obtain an approximation of the solution of the Hammerstein equation in the space $\mathbf{X}_{k}$ by solving (3.9) exactly to obtain the solution $z_{k}$. The next step is to obtain an approximation of the solution $z_{k+1}$ of (3.9) with $n=k+1$. For this purpose, we decompose

$$
z_{k+1}=z_{k+1}^{L}+z_{k+1}^{H}, \quad \text { with } z_{k+1}^{L} \in \mathbf{X}_{k} \text { and } z_{k+1}^{H} \in \mathbf{W}_{k+1}
$$

so that (3.9) becomes

$$
z_{k+1}^{L}+z_{k+1}^{H}=P_{k+1} \Psi\left(f+K z_{k+1}\right) .
$$


This equation can be transformed into

$$
z_{k+1}^{L}=P_{k} \Psi\left(f+K z_{k+1}\right)+\left(P_{k+1}-P_{k}\right) \Psi\left(f+K z_{k+1}\right)-z_{k+1}^{H}
$$

or, equivalently,

$$
P_{k}\left(z_{k+1}^{L}+z_{k+1}^{H}\right)=P_{k} \Psi\left(f+K z_{k+1}\right)+\left(P_{k+1}-P_{k}\right) \Psi\left(f+K z_{k+1}\right)-z_{k+1}^{H} .
$$

Here we used the fact that $P_{k} z_{k+1}^{H}=0$ as $z_{k+1}^{H} \in \mathbf{W}_{k+1}$, and $P_{k}$ is a projection onto $\mathbf{X}_{k}$. Next, we compute

$$
z_{k, 1}^{H}:=\left(P_{k+1}-P_{k}\right) \Psi\left(f+K z_{k}\right)
$$

and note that $z_{k, 1}^{H} \in \mathbf{W}_{k+1}$. In (4.1), we replace both $z_{k+1}^{H}$ and the second term on the right-hand side by $z_{k, 1}^{H}$ to obtain an equation for $z_{k, 1}^{L} \in \mathbf{X}_{k}$ :

$$
z_{k, 1}^{L}=P_{k} \Psi\left(f+K\left(z_{k, 1}^{L}+z_{k, 1}^{H}\right)\right) .
$$

The element $z_{k, 1}^{L}$ turns out to be a good approximation to $z_{k+1}^{L}$. We then obtain an approximation to the solution $z_{k+1}$ of (3.9) by setting

$$
z_{k, 1}:=z_{k, 1}^{L}+z_{k, 1}^{H} \text {. }
$$

Note that $z_{k, 1}^{L}$ and $z_{k, 1}^{H}$, respectively, represent the lower and higher frequency components of $z_{k, 1}$.

We continue this process to find an approximation of the solution of (3.9) with $n=k+2$. Specifically, we compute

$$
z_{k, 2}^{H}:=\left(P_{k+2}-P_{k}\right) \Psi\left(f+K z_{k, 1}\right)
$$

using the approximate solution $z_{k, 1}$ obtained in the previous step, and solve for $z_{k, 2}^{L} \in$ $\mathbf{X}_{k}$ from

$$
z_{k, 2}^{L}=P_{k} \Psi\left(f+K\left(z_{k, 2}^{L}+z_{k, 2}^{H}\right)\right) .
$$

An approximation to the solution $z_{k+2}$ of (3.9) with $n=k+2$ is hence obtained by setting

$$
z_{k, 2}:=z_{k, 2}^{L}+z_{k, 2}^{H} .
$$

This procedure is repeated to obtain an approximation $z_{k, m}$ of the solution $z_{k+m}$ of (3.9) with $n=k+m$. Once $z_{k, m}$ is obtained, let

$$
u_{k, m}=f+K z_{k, m}
$$

which approximates the solution $u$ of (3.4), as with $n=k+m, u_{n}(t)=f(t)+$ $\int_{0}^{1} k(t, s) z_{n}(s) d s$. The steps just discussed can be summarized in the following algorithm.

Algorithm 1. The multilevel augmentation method: An operator form.

Let $k$ be a fixed positive integer.

Step 1. Find the solution $z_{k} \in \mathbf{X}_{k}$ of (3.9) with $n:=k$. Set $z_{k, 0}:=z_{k}$ and $\ell:=1$.

Step 2. Compute

$$
z_{k, \ell}^{H}:=\left(P_{k+l}-P_{k}\right) \Psi\left(f+K z_{k, \ell-1}\right) .
$$

Copyright (c) by SIAM. Unauthorized reproduction of this article is prohibited. 
Step 3. Obtain $z_{k, \ell}^{L} \in \mathbf{X}_{k}$ as the solution of

$$
z_{k, \ell}^{L}=P_{k} \Psi\left(f+K\left(z_{k, \ell}^{L}+z_{k, \ell}^{H}\right)\right) .
$$

Step 4. Let

$$
z_{k, \ell}:=z_{k, \ell}^{L}+z_{k, \ell}^{H} .
$$

Set $\ell \leftarrow \ell+1$ and go back to Step 2 until $\ell=m$.

Step 5. Obtain the approximate solution of $u_{n}$ in (3.4) by $u_{k, m}:=f+K z_{k, m}$.

The existence of the solution $z_{k, m}^{L}$ of (4.8) can be guaranteed similarly as in Theorem 3.1. Let

$$
S(a)(u)(t):=f(t)+K(u+a)(t), \quad u, a \in L^{\infty}(I),
$$

so that (4.8) with $\ell=m$ can be written as

$$
z_{k, m}^{L}=P_{k} \Psi S\left(z_{k, m}^{H}\right)\left(z_{k, m}^{L}\right) .
$$

The following is similar to Theorem 3.1 and can be viewed as its corollary.

Corollary 4.1. Let $z$ be an isolated solution of (3.6). Assume that 1 is not an eigenvalue of the linear operator $(\Psi S(a))^{\prime}(z)$, where $(\Psi S(a))^{\prime}(z)$ denotes the Fréchet derivative of $\Psi S(a)$ at $z$ with $a \in L^{\infty}(I)$. Then (4.10) has a unique solution $z_{k, m}^{L}$ in $a$ ball $B(z, \delta):=\left\{c \in C(I):\|x-z\|_{\infty} \leq \delta\right\}$ for some $\delta>0$ and for sufficiently large $k$. Moreover, there exists a constant $0<q<1$, independent of $k$, such that

$$
\frac{\alpha_{k}}{1+q} \leq\left\|z_{k, m}^{L}-z\right\|_{\infty} \leq \frac{\alpha_{k}}{1-q}
$$

where $\alpha_{n}:=\left\|\left(I-\left(P_{n} \Psi S(a)\right)^{\prime}(z)\right)^{-1}\left(\left(P_{k} \Psi S(a)\right)(z)-\Psi S(a)(z)\right)\right\|_{\infty}$. Finally

$$
E_{k}(z) \leq\left\|z_{k, m}^{L}-z\right\|_{\infty} \leq C E_{k}(z)
$$

where $C$ is a constant independent of $k$ and $E_{k}(z)=\inf _{u \in \mathbf{X}_{k}}\|z-u\|_{\infty}$.

The following lemma is analogous to Lemma 3.2 of [8].

Lemma 4.2. Let $z$ be an isolated solution of (3.6). Assume that 1 is not an eigenvalue of $(\Psi S)^{\prime}(z)$. Then there exists a sequence of positive numbers $\alpha_{k, m}, k \in \mathbf{N}$, $m \in \mathbf{N}_{0}$ with $\lim _{k \rightarrow \infty} \alpha_{k, m}=0$ uniformly for $m \in \mathbf{N}_{0}$ and a positive integer $N$ such that for all $k \geq N$ and $m \in \mathbf{N}_{0}$,

$$
\left\|z_{k, m}-z_{k+m}\right\|_{\infty} \leq \alpha_{k, m}\left\|z_{k, m-1}-z_{k+m}\right\|_{\infty} .
$$

Proof. From (4.7), (4.8), and (4.9),

$$
\begin{aligned}
z_{k, m} & =z_{k, m}^{H}+z_{k, m}^{L} \\
& =\left(P_{k+m}-P_{k}\right) \Psi\left(f+K z_{k, m-1}\right)+P_{k} \Psi\left(f+K z_{k, m}\right) .
\end{aligned}
$$

Using (4.11) and (3.9) with $n=k+m$,

$$
\begin{aligned}
& \left(I-(\Psi S)^{\prime}\left(z_{k+m}+\theta_{1}\left(z_{k+m}-z_{k, m}\right)\right)\right)\left(z_{k, m}-z_{k+m}\right) \\
& \quad=\left(P_{k+m}-P_{k}\right)\left(\Psi S\left(z_{k, m-1}\right)-\Psi S\left(z_{k+m}\right)\right) \\
& \quad=\left(P_{k+m}-P_{k}\right)(\Psi S)^{\prime}\left(z_{k+m}+\theta_{2}\left(z_{k+m}-z_{k, m-1}\right)\right)\left(z_{k+m}-z_{k, m-1}\right),
\end{aligned}
$$

Copyright $@$ ㅇ by SIAM. Unauthorized reproduction of this article is prohibited. 
where $0<\theta_{1}, \theta_{2}<1$. Let $\alpha_{k, m}:=\gamma\left\|\left(P_{k+m}-P_{k}\right)(\Psi S)^{\prime}\left(z_{k+m}+\theta_{2}\left(z_{k+m}-z_{k, m-1}\right)\right)\right\|_{\infty}$, where $\left\|I-(\Psi S)^{\prime}\left(z_{k+m}+\theta_{1}\left(z_{k+m}-z_{k, m}\right)\right)\right\|_{\infty}^{-1}<\gamma$ for all $k, m$. Assumptions (A1), (A2), and (A5) guarantee that $\alpha_{k, m} \rightarrow 0$ uniformly in $m \in \mathbf{N}_{0}$ as $k \rightarrow \infty$, and finally,

$$
\left\|z_{k, m}-z_{k+m}\right\|_{\infty} \leq \alpha_{k, m}\left\|z_{k, m-1}-z_{k+m}\right\|_{\infty} .
$$

The rate of convergence of the multilevel augmentation method is now discussed. We utilize the idea of a majorization sequence introduced in [8]. A sequence of nonnegative numbers $\gamma_{n}, n \in \mathbf{N}_{0}$, is called a majorization sequence of $E_{n}, n \in \mathbf{N}_{0}$, if $\gamma_{n} \geq E_{n}$ for all $n \in \mathbf{N}_{0}$ and there exist a positive integer $N_{0}$ and a positive constant $\sigma$ such that for $n \geq N_{0}$,

$$
\frac{\gamma_{n+1}}{\gamma_{n}} \geq \sigma
$$

For the wavelet collocation method described in section 3 , it is known by Theorem 5.2 of [8], which describes a multilevel augmentation method based upon the wavelet collocation method, that the majorization constant can be selected as

$$
\gamma_{n}:=c \frac{1}{2^{r n}}\|z\|_{r, 2},
$$

where $z \in W_{\kappa, 2}([0,1]), \kappa$ is the order of the wavelet and $c$ is independent of $n$ such that $E_{n} \leq \gamma_{n}$. Proof of the following theorem is included for completeness (see Theorem 3.3 in $[8])$.

THEOREM 4.3. Let $z$ be an isolated solution of (3.6), and let $\gamma_{n}, n \in \mathbf{N}_{0}$, be a majorization sequence of $E_{n}, n \in \mathbf{N}_{0}$. Assume that 1 is not an eigenvalue of $(\Psi S)^{\prime}(z)$. Then there exist a positive constant $\rho$ and a positive constant $N$ such that for all $k \geq N$ and $m \in \mathbf{N}_{0}$,

$$
\left\|z-z_{k, m}\right\|_{\infty} \leq(\rho+1) \gamma_{k+m} .
$$

Proof. We apply induction on $m$. The estimate clearly holds with $m=0$. Suppose that (4.12) holds for $m-1$. Then, using the inductive hypothesis and the majorization property,

$$
\begin{aligned}
\left\|z_{k, m-1}-z_{k+m}\right\|_{\infty} & \leq\left\|z_{k, m-1}-z\right\|_{\infty}+\left\|z_{k+m}-z\right\|_{\infty} \\
& \leq\left(\rho+\frac{\rho+1}{\sigma}\right) \gamma_{k+m} .
\end{aligned}
$$

Choosing $N$ large enough that for $k>N$, Lemma 3.2 holds and that $\alpha_{k, m}\left(\rho+\frac{\rho+1}{\sigma}\right)<$ 1 , we get

$$
\left\|z_{k, m}-z_{k+m}\right\|_{\infty} \leq \gamma_{k+m}
$$

Finally,

$$
\left\|z_{k, m}-z\right\|_{\infty} \leq\left\|z_{k, m}-z_{k+m}\right\|_{\infty}+\left\|z_{k+m}-z\right\|_{\infty} \leq(\rho+1) \gamma_{k+m} .
$$

4.1. Discrete multilevel augmentation method for Hammerstein equation. In this subsection, a discrete version of the multilevel augmentation method is described. For any $v \in \mathbf{X}_{k+m}$, we have a unique expansion

$$
v=\sum_{(i, j) \in U_{k+m}} v_{i, j} w_{i, j} .
$$

Copyright $@$ by SIAM. Unauthorized reproduction of this article is prohibited. 
The vector $\mathbf{v}:=\left[v_{i, j}:(i, j) \in U_{k+m}\right]^{T}$ represents $v$. Thus, for $z_{k, m}$, its representation vector is given by $\mathbf{z}_{k, m}:=\left[\left(z_{k, m}\right)_{i, j}:(i, j) \in U_{k+m}\right]^{T}$. Setting $U_{k, m}:=U_{k+m} \backslash U_{k}$, we obtain that

$$
U_{k, m}=\left\{(i, j): j \in Z_{w(i)}, i \in Z_{k+m+1} \backslash Z_{k+1}\right\} .
$$

Consequently, we have the representations

$$
z_{k, m}^{L}:=\sum_{(i, j) \in U_{k}}\left(\mathbf{z}_{k, m}\right)_{i, j} w_{i, j} \quad \text { and } \quad z_{k, m}^{H}:=\sum_{(i, j) \in U_{k, m}}\left(\mathbf{z}_{k, m}\right)_{i, j} w_{i, j} .
$$

Algorithm 2. The fast multilevel augmentation method: A discrete form.

Let $k$ be a fixed positive integer.

Step 1. Apply the fast wavelet collocation method described in section 3 to solve the nonlinear system

$$
\begin{aligned}
& \left\langle\ell_{i^{\prime}, j^{\prime}}, \sum_{(i, j) \in U_{k}}\left(\mathbf{z}_{k}\right)_{i, j} w_{i, j}\right\rangle \\
& =\left\langle\ell_{i^{\prime}, j^{\prime}}, \Psi\left(f+K\left(\sum_{(i, j) \in U_{k}}\left(\mathbf{z}_{k}\right)_{i, j} w_{i, j}\right)\right)\right\rangle
\end{aligned}
$$

for $\left(i^{\prime}, j^{\prime}\right) \in U_{k}$, and for $\mathbf{z}_{k}:=\left[\left(\mathbf{z}_{k}\right)_{i, j}:(i, j) \in U_{k}\right]^{T}$.

Let $z_{k, 0}:=\sum_{(i, j) \in U_{k}}\left(\mathbf{z}_{k}\right)_{i, j} w_{i, j}$ and $\ell:=1$.

Step 2. Solve the linear system

$$
\left\langle\ell_{i^{\prime}, j^{\prime}}, \sum_{(i, j) \in U_{k, \ell}}\left(\mathbf{z}_{k, \ell}^{h}\right)_{i, j} w_{i, j}\right\rangle=\left\langle\ell_{i^{\prime}, j^{\prime}}, \Psi\left(f+K z_{k, \ell-1}\right)\right\rangle
$$

for $\left(i^{\prime}, j^{\prime}\right) \in U_{k, \ell}$ to obtain $\mathbf{z}_{k, \ell}^{h}$, and define $z_{k, \ell}^{H}:=\sum_{(i, j) \in U_{k, \ell}}\left(\mathbf{z}_{k, \ell}^{h}\right)_{i, j} w_{i, j}$.

Step 3. Solve the nonlinear system with the fast wavelet collocation strategy

$$
\begin{aligned}
& \left\langle\ell_{i^{\prime}, j^{\prime}}, \sum_{(i, j) \in U_{k}}\left(\mathbf{z}_{k, \ell}^{l}\right)_{i, j} w_{i, j}\right\rangle \\
& =\left\langle\ell_{i^{\prime}, j^{\prime}}, \Psi\left(f+K\left(\sum_{(i, j) \in U_{k}}\left(\mathbf{z}_{k, l}^{l}\right)_{i, j} w_{i, j}+z_{k, l}^{H}\right)\right)\right\rangle
\end{aligned}
$$

for $\left(i^{\prime}, j^{\prime}\right) \in U_{k}$ to obtain $\mathbf{z}_{k, \ell}^{\ell}$ and $z_{k, \ell}^{L}:=\sum_{(i, j) \in U_{k, \ell}}\left(\mathbf{z}_{k, \ell}^{\ell}\right)_{i, j} w_{i, j}$ and

$$
z_{k, \ell}=z_{k, \ell}^{L}+z_{k, \ell}^{H} .
$$

Step 4. Set $\ell \leftarrow \ell+1$ and go back to Step 2 until $\ell=m$.

Step 5. Obtain the approximation solution of $u_{n}, n=k+m$, from

$$
u_{n}:=f+K z_{k, m} .
$$

Copyright $@$ by SIAM. Unauthorized reproduction of this article is prohibited. 
We make several comments regarding Algorithm 2. First of all, we note that (4.7) is equivalent to (4.14). This follows from the fact that (4.7) is equivalent to

$$
\left(P_{k+\ell}-P_{k}\right)\left(z_{k, \ell}^{H}-\Psi\left(f+K z_{k, \ell-1}\right)\right)=0,
$$

and this equation is equivalent to (4.14) on the basis of Theorem 4.2 of [8] as $\mathbf{V}_{p} \subset \mathbf{X}_{k}^{\perp}$, $p>k$. Here recall $\mathbf{V}_{i}:=\operatorname{span}\left\{\ell_{i, j}: j \in Z_{\omega(i)}\right\}$. For solving the nonlinear equations (4.13) and (4.15), we employed the fast wavelet collocation method for Hammerstein equations which is described in (3.22)-(3.23). Now, we count the number of multiplications used in Algorithm 2 as a measure for complexity of the computation, a standard used in [8]. In the cost analysis of the discrete form of the multilevel augmentation method (Algorithm 2) of [8], it is assumed that the initial approximate solution $u_{k}$ has been obtained and the same Jacobian matrix is used throughout the computation. Despite the absence of updating the Jacobian at each step, it is reported in numerical examples [8] that accurate approximations are obtained at the expense of increasing the number of iterations. Recall that we observed the same phenomenon in Examples 3.8 and 3.9. In this paper, the total computational cost obtained below includes the computational cost of obtaining $u_{k}$ from (4.13) and that of solving the nonlinear system (4.15) with the Jacobian being updated at each step of the iteration. For $(i, j),\left(i^{\prime}, j^{\prime}\right) \in U_{k, \ell}$, let $\mathbf{E}_{k, \ell}:=\left[\left\langle\ell_{i^{\prime} j^{\prime}}, w_{i j}\right\rangle:(i, j),\left(i^{\prime}, j^{\prime}\right) \in U_{k, \ell}\right]$, $\mathbf{f}_{k, \ell}:=\left[\left\langle\ell_{i^{\prime}, j^{\prime}}, \Psi\left(f+K z_{k, \ell-1}\right)\right\rangle:\left(i^{\prime}, j^{\prime}\right) \in U_{k, \ell}\right]^{T}$, and $\mathbf{z}_{k, \ell}^{H}:=\left[\left(z_{k, \ell}^{h}\right)_{i j}:(i, j) \in U_{k, \ell}\right]$. Here it is assumed that the integrals that appear in $\mathbf{f}_{k, \ell}$ require a constant computational cost per integral. Equation (4.14) can be written as

$$
\mathbf{E}_{k, \ell} \mathbf{z}_{k, \ell}^{H}=\mathbf{f}_{k, \ell} .
$$

For each stage $i$ with $i=1,2, \ldots, m$, to execute Algorithm 2, the following procedures must be performed:

(1) Generate the coefficient matrix $\mathbf{E}_{k, \ell}$.

(2) Compute the vector $\mathbf{f}_{k, \ell}$.

(3) Solve the linear system (4.16).

(4) Solve the nonlinear system (4.15).

As in [8], we denote by $\mathcal{M}_{k, i, j}$ the computational cost in the procedure $j$ at stage $i$. Recall $f(i)=\operatorname{dim}\left(\mathbf{X}_{i}\right)=\kappa \mu^{i}$.

Lemma 4.4. For any $i>0$, with $f(i)=\operatorname{dim}\left(\mathbf{X}_{i}\right), \mathcal{M}_{k, i, 2}=\mathcal{O}(f(k+i)), \mathcal{M}_{k, i, j}=$ $\mathcal{O}(f(k+i) \log f(k+i)), j=1,3$, and $\mathcal{M}_{k, i, 4}=\mathcal{O}(f(k) \log f(k))$.

Proof. For $\mathcal{M}_{k, i, j}, j=1,2,3$, the results follow from Proposition 4.3 of [8]. For $j=4$, we note that application of the fast wavelet collocation method reduces the number of nonzero entries in the Jacobian to $\mathcal{O}(f(k) \log f(k))$ by Lemma 3.3.

Now let $\overline{\mathcal{M}}_{k, m, j}$ denote the total computational cost associated with procedure $j$ in obtaining the solution $z_{k, m}$.

LemMa 4.5. $\overline{\mathcal{M}}_{k, i, 2}=\mathcal{O}(f(k+m) \log f(k+m)), \overline{\mathcal{M}}_{k, i, j}=\mathcal{O}(f(k+m)), j=1,3$, and $\overline{\mathcal{M}}_{k, i, 4}=\mathcal{O}(f(k) \log f(k))$.

Proof. For $j=1,2,3,4, \overline{\mathcal{M}}_{k, m, j}=\sum_{i=1}^{m} \mathcal{M}_{k, i, j}$. This lemma is thus proved by Lemma 4.4 and by noting $f(k)=\kappa \mu^{k}$.

THEOREM 4.6. The total computational cost in obtaining the solution $u_{k, m}$ by Algorithm 2 is of the order $\mathcal{O}(f(k+m) \log f(k+m))$.

Proof. Since the total computational cost in obtaining $u_{k, m}$ is $\sum_{j=1}^{4} \overline{\mathcal{M}}_{k, m, j}$, noting that $f(k+m)=\mathcal{O}\left(\mu^{k+m}\right)$, the proof is complete by Lemma 4.5.

It should be noted that the estimate for the computational cost described in Theorem 4.6 is obtained under the assumption (see assumption (A0), page 2333 of [8]) 
that "computing the integrals that appear in $\mathbf{f}_{k, \ell}$ requires a constant computational cost per integral." As was pointed out also at the end of section 5 of [8], in actual computation, additional computational efforts arise in computing $\mathbf{f}_{k, \ell}$ due to the influence of numerical integration. For the one dimensional case, the total computational cost is $\mathcal{O}\left(f(k+m)(\log f(k+m))^{3}\right)$. See [8] for more details. This cost is reflected in the tables for numerical examples below.

4.2. Numerical results. Numerical examples for the multilevel augmentation methods to solve the Hammerstein equation are presented below. We let $e_{h}^{\prime}:=$ $\left\|u-u_{k, m}\right\|_{0, \infty}, R_{h}^{\prime}:=\log _{2}\left(\frac{e_{h}^{\prime}}{e_{h / 2}^{\prime}}\right)$, where $u_{k, m}$ is the multilevel augmentation solution with fixed $k$. As was the case with Example 3.8, we have tested the fast multilevel augmentation method on equations given below in Examples 4.7, 4.8, 4.9, and 4.10, where condition (3.14) is relaxed. Results obtained by setting $M=2$ are excellent. Here we use the prime symbol ' to represent the multilevel augmentation results.

Example 4.7. Consider the equation

$$
u(t)-\int_{0}^{1} \sin (\pi(s+t)) u^{2}(s) d s=f(t), \quad t \in[0,1],
$$

where $f(t)$ is chosen so that the isolated solution $u(t)=\sin (\pi t)$.

Example 4.8. Consider the equation

$$
u(t)-\int_{0}^{1} \log |s-t| \cos (u(s)) d s=f(t), \quad t \in[0,1],
$$

where $f(t)$ is chosen so that the isolated solution $u(t)=\arccos \left(t^{2}\right)$.

From Examples 4.7 and 4.8, we see that the computing time of the fast multilevel augmentation solutions is far less than the computing time of the full collocation solutions when the quasi-Newton method is used, and the reduction is even more pronounced when the Newton's method is used. Similar reductions in computing times can be observed for weakly singular equation in Example 4.9, albeit somewhat milder in the order of reduction. Therefore, the fast multilevel augmentation method, while preserving the numerical accuracy, provides the fastest numerical scheme for Hammerstein equations among the four methods we have tested. It is interesting to observe also from Tables 6 and 7 that application of the quasi-Newton method does not improve the computing time when it is used in conjunction with the multilevel augmentation methods.

Example 4.9. Consider the equation

$$
u(t)-\int_{0}^{1} \log |s-t| u^{2}(s) d s=f(t), \quad t \in[0,1],
$$

where $f(t)$ is chosen so that the isolated solution $u(t)=t^{3 / 2}$. Table 8 contains numerical results.

Example 4.10. Consider the equation

$$
u(t)-\int_{0}^{1} \sin (s+t) \arctan (u(s)) d s=f(t), \quad t \in[0,1],
$$

where $f(t)$ is chosen so that the isolated solution $u(t)=\exp (t)$. Table 9 contains numerical results. 
TABLE 6

Computational results for the Hammerstein equation for Example 4.7.

\begin{tabular}{|c|c|c|c|c|c|c|c|c|}
\hline \multirow{2}{*}{$f(n)$} & \multicolumn{3}{|c|}{ Full collocation solution } & \multicolumn{4}{|c|}{ Fast collocation solution } \\
\cline { 2 - 9 } & $e_{h}$ & $R_{h}$ & $T_{N}$ & $T_{Q}$ & $\widetilde{e}_{h}$ & $\widetilde{R}_{h}$ & $\widetilde{T}_{N}$ & $\widetilde{T}_{Q}$ \\
\hline 16 & $3.1193 \mathrm{e}-3$ & & 0.13 & 0.10 & $3.1193 \mathrm{e}-3$ & & 0.13 & 0.10 \\
32 & $7.7377 \mathrm{e}-4$ & 2.01 & 0.47 & 0.30 & $7.7338 \mathrm{e}-4$ & 2.01 & 0.46 & 0.27 \\
64 & $1.9307 \mathrm{e}-4$ & 2.00 & 1.67 & 1.12 & $1.9229 \mathrm{e}-4$ & 2.01 & 1.64 & 0.89 \\
128 & $4.8244 \mathrm{e}-5$ & 2.00 & 7.48 & 4.17 & $4.7375 \mathrm{e}-5$ & 2.02 & 5.97 & 2.73 \\
256 & $1.2059 \mathrm{e}-5$ & 2.00 & 30.06 & 16.48 & $1.1178 \mathrm{e}-5$ & 2.08 & 22.22 & 8.63 \\
512 & $3.0147 \mathrm{e}-6$ & 2.00 & 120.84 & 68.59 & $2.1673 \mathrm{e}-6$ & 2.37 & 83.84 & 31.54 \\
\hline
\end{tabular}

\begin{tabular}{|c|c|c|c|c|c|c|c|c|c|}
\hline \multicolumn{10}{|c|}{ Multilevel augmentation schemes when $k=3$} \\
\hline \multirow{2}{*}{$m$} & \multirow{2}{*}{$f(n)$} & \multicolumn{3}{|c|}{ Full multilevel solution } & \multicolumn{3}{|c|}{ Fast multilevel solution } \\
\cline { 3 - 10 } & & $e_{h}^{\prime}$ & $R_{h}^{\prime}$ & $T_{N}^{\prime}$ & $T_{Q}^{\prime}$ & $\widetilde{e}_{h}^{\prime}$ & $\widetilde{R}_{h}^{\prime}$ & $\widetilde{T}_{N}^{\prime}$ & $\widetilde{T}_{Q}^{\prime}$ \\
\hline 0 & 16 & $3.1193 \mathrm{e}-3$ & & 0.17 & 0.12 & $3.1193 \mathrm{e}-3$ & & 0.17 & 0.13 \\
1 & 32 & $7.4746 \mathrm{e}-4$ & 2.06 & 0.39 & 0.29 & $7.4710 \mathrm{e}-4$ & 2.06 & 0.36 & 0.28 \\
2 & 64 & $1.8534 \mathrm{e}-4$ & 2.01 & 1.06 & 0.91 & $1.8456 \mathrm{e}-4$ & 2.02 & 0.79 & 0.69 \\
3 & 128 & $4.6238 \mathrm{e}-5$ & 2.00 & 3.37 & 3.17 & $4.5381 \mathrm{e}-5$ & 2.02 & 1.83 & 1.79 \\
4 & 256 & $1.1554 \mathrm{e}-5$ & 2.00 & 12.36 & 12.09 & $1.0683 \mathrm{e}-5$ & 2.09 & 4.47 & 4.30 \\
5 & 512 & $2.8880 \mathrm{e}-6$ & 2.00 & 47.83 & 47.65 & $2.0555 \mathrm{e}-6$ & 2.38 & 11.45 & 11.16 \\
\hline
\end{tabular}

TABLE 7

Computational results for the Hammerstein equation for Example 4.10.

\begin{tabular}{|c|c|c|c|c|c|c|c|c|}
\hline \multirow{2}{*}{$f(n)$} & \multicolumn{3}{|c|}{ Full collocation solution } & \multicolumn{4}{c|}{ Fast collocation solution } \\
\cline { 2 - 9 } & $e_{h}$ & $R_{h}$ & $T_{N}$ & $T_{Q}$ & $\widetilde{e}_{h}$ & $\widetilde{R}_{h}$ & $\widetilde{T}_{N}$ & $\widetilde{T}_{Q}$ \\
\hline 16 & $9.4243 \mathrm{e}-5$ & & 3.06 & 2.07 & $9.4243 \mathrm{e}-5$ & & 2.92 & 1.68 \\
32 & $2.3555 \mathrm{e}-5$ & 2.00 & 11.52 & 6.00 & $2.3555 \mathrm{e}-5$ & 2.00 & 11.17 & 5.91 \\
64 & $5.8883 \mathrm{e}-6$ & 2.00 & 46.39 & 25.29 & $5.8883 \mathrm{e}-6$ & 2.00 & 44.22 & 24.31 \\
128 & $1.4720 \mathrm{e}-6$ & 2.00 & 235.41 & 134.83 & $1.4720 \mathrm{e}-6$ & 2.00 & 175.90 & 118.08 \\
256 & $3.6798 \mathrm{e}-7$ & 2.00 & 1028.88 & 522.518 & $3.6800 \mathrm{e}-7$ & 2.00 & 699.15 & 518.63 \\
512 & $9.1972 \mathrm{e}-8$ & 2.00 & 3672.00 & 2268.07 & $9.1990 \mathrm{e}-8$ & 2.00 & 2807.65 & 2046.92 \\
\hline
\end{tabular}

\begin{tabular}{|c|c|c|c|c|c|c|c|c|c|}
\hline \multicolumn{10}{|c|}{ Multilevel augmentation schemes when $k=3$} \\
\hline \multirow{2}{*}{$m$} & \multirow{2}{*}{$f(n)$} & \multicolumn{3}{|c|}{ Full multilevel solution } & \multicolumn{3}{c|}{ Fast multilevel solution } \\
\cline { 3 - 10 } & & $e_{h}^{\prime}$ & $R_{h}^{\prime}$ & $T_{N}^{\prime}$ & $T_{Q}^{\prime}$ & $\widetilde{e}_{h}^{\prime}$ & $\widetilde{R}_{h}^{\prime}$ & $\widetilde{T}_{N}^{\prime}$ & $\widetilde{T}_{Q}^{\prime}$ \\
\hline 0 & 16 & $9.4243 \mathrm{e}-5$ & & 3.08 & 1.77 & $9.4243 \mathrm{e}-5$ & & 2.91 & 1.74 \\
1 & 32 & $2.3546 \mathrm{e}-5$ & 2.00 & 6.19 & 3.55 & $2.3546 \mathrm{e}-5$ & 2.00 & 5.85 & 3.49 \\
2 & 64 & $5.8855 \mathrm{e}-6$ & 2.00 & 9.50 & 5.63 & $5.8883 \mathrm{e}-6$ & 2.00 & 9.03 & 5.45 \\
3 & 128 & $1.4713 \mathrm{e}-6$ & 2.00 & 13.67 & 8.53 & $1.4720 \mathrm{e}-6$ & 2.00 & 12.58 & 7.77 \\
4 & 256 & $3.6780 \mathrm{e}-7$ & 2.00 & 21.06 & 14.67 & $3.6781 \mathrm{e}-7$ & 2.00 & 17.12 & 11.09 \\
5 & 512 & $9.1925 \mathrm{e}-8$ & 2.00 & 41.02 & 33.36 & $9.1943 \mathrm{e}-8$ & 2.00 & 24.01 & 16.80 \\
\hline
\end{tabular}

5. Conclusions. In this paper, we introduced a wavelet collocation method as well as a multilevel augmentation method for nonlinear Hammerstein equations. It was shown that the computational complexity can be reduced from $\mathcal{O}\left(N^{2}\right)$ to $\mathcal{O}(N \log N)$. This replicates the order of reduction which was recently achieved in relation to the computation in approximating the solution of the Fredholm equation of the second kind [21], [7], [9]. The reduction was made possible by "linearizing" the Hammerstein equation to take full advantage of the properties which wavelet basis functions provide, namely the property of the vanishing moments as well as that of the supports being separated from the diagonal. An application of the wavelets results in a fast algorithm due to the sparse structures of the Jacobian matrices, which are induced by the block truncation strategy. For those methods discussed in [2], computational complexities are at least of order $\mathcal{O}\left(N^{2}\right)$. Hence, the fast wavelet collocation method for nonlinear Hammerstein equations presented in this paper outperforms 
TABLE 8

Computational results for the Hammerstein equation for Example 4.8.

\begin{tabular}{|c|c|c|c|c|c|c|c|c|}
\hline \multirow{2}{*}{$f(n)$} & \multicolumn{3}{|c|}{ Full collocation solution } & \multicolumn{4}{|c|}{ Fast collocation solution } \\
\cline { 2 - 9 } & $e_{h}$ & $R_{h}$ & $T_{N}$ & $T_{Q}$ & $\widetilde{e}_{h}$ & $\widetilde{R}_{h}$ & $\widetilde{T}_{N}$ & $\widetilde{T}_{Q}$ \\
\hline 16 & $3.8625 \mathrm{e}-3$ & & 0.07 & 0.04 & $3.8625 \mathrm{e}-3$ & & 0.13 & 0.06 \\
32 & $9.9128 \mathrm{e}-4$ & 1.96 & 0.27 & 0.14 & $9.9130 \mathrm{e}-4$ & 1.96 & 0.27 & 0.14 \\
64 & $2.5050 \mathrm{e}-4$ & 1.98 & 1.06 & 0.56 & $2.5054 \mathrm{e}-4$ & 1.98 & 1.02 & 0.50 \\
128 & $6.2908 \mathrm{e}-5$ & 1.99 & 4.23 & 2.20 & $6.2959 \mathrm{e}-5$ & 1.99 & 3.94 & 1.82 \\
256 & $1.5761 \mathrm{e}-5$ & 2.00 & 17.20 & 9.18 & $1.5813 \mathrm{e}-5$ & 1.99 & 15.44 & 6.97 \\
512 & $3.9456 \mathrm{e}-6$ & 2.00 & 68.95 & 35.88 & $3.9985 \mathrm{e}-6$ & 1.98 & 59.91 & 26.68 \\
\hline
\end{tabular}

\begin{tabular}{|c|c|c|c|c|c|c|c|c|c|}
\hline \multicolumn{10}{|c|}{ Multilevel augmentation schemes when $k=3$} \\
\hline \multirow{2}{*}{$m$} & \multirow{2}{*}{$f(n)$} & \multicolumn{3}{|c|}{ Full multilevel solution } & \multicolumn{3}{|c|}{ Fast multilevel solution } \\
\cline { 3 - 10 } & & $e_{h}^{\prime}$ & $R_{h}^{\prime}$ & $T_{N}^{\prime}$ & $T_{Q}^{\prime}$ & $\widetilde{e}_{h}^{\prime}$ & $\widetilde{R}_{h}^{\prime}$ & $\widetilde{T}_{N}^{\prime}$ & $\widetilde{T}_{Q}^{\prime}$ \\
\hline 0 & 16 & $3.8625 \mathrm{e}-3$ & & 0.08 & 0.04 & $3.8625 \mathrm{e}-3$ & & 0.07 & 0.04 \\
1 & 32 & $1.1792 \mathrm{e}-3$ & 1.71 & 0.18 & 0.11 & $1.7367 \mathrm{e}-3$ & 1.71 & 0.18 & 0.11 \\
2 & 64 & $2.9148 \mathrm{e}-4$ & 2.02 & 0.38 & 0.30 & $2.9152 \mathrm{e}-4$ & 2.02 & 0.33 & 0.27 \\
3 & 128 & $6.9380 \mathrm{e}-5$ & 2.07 & 1.01 & 0.93 & $6.9428 \mathrm{e}-5$ & 2.07 & 0.65 & 0.57 \\
4 & 256 & $1.6776 \mathrm{e}-5$ & 2.05 & 3.53 & 3.39 & $1.6828 \mathrm{e}-5$ & 2.04 & 1.63 & 1.50 \\
5 & 512 & $4.1271 \mathrm{e}-6$ & 2.02 & 17.08 & 16.89 & $4.1800 \mathrm{e}-6$ & 2.01 & 7.67 & 7.40 \\
\hline
\end{tabular}

TABLE 9

Computational results for the Hammerstein equation for Example 4.9.

\begin{tabular}{|c|c|c|c|c|c|c|c|c|}
\hline \multirow{2}{*}{$f(n)$} & \multicolumn{3}{|c|}{ Full collocation solution } & \multicolumn{4}{|c|}{ Fast collocation solution } \\
\cline { 2 - 9 } & $e_{h}$ & $R_{h}$ & $T_{N}$ & $T_{Q}$ & $\widetilde{e}_{h}$ & $\widetilde{R}_{h}$ & $\widetilde{T}_{N}$ & $\widetilde{T}_{Q}$ \\
\hline 16 & $1.3394 \mathrm{e}-3$ & & 0.16 & 0.10 & $1.3394 \mathrm{e}-3$ & & 0.16 & 0.10 \\
32 & $2.9270 \mathrm{e}-4$ & 2.19 & 0.62 & 0.29 & $2.9269 \mathrm{e}-4$ & 2.19 & 0.61 & 0.31 \\
64 & $6.7835 \mathrm{e}-5$ & 2.11 & 2.43 & 1.00 & $6.7827 \mathrm{e}-5$ & 2.11 & 2.30 & 0.87 \\
128 & $1.6329 \mathrm{e}-5$ & 2.05 & 9.69 & 3.72 & $1.6317 \mathrm{e}-5$ & 2.05 & 8.80 & 2.85 \\
256 & $4.0185 \mathrm{e}-6$ & 2.02 & 38.79 & 14.74 & $4.0072 \mathrm{e}-6$ & 2.02 & 34.07 & 9.82 \\
512 & $9.9604 \mathrm{e}-7$ & 2.01 & 155.76 & 59.88 & $9.8410 \mathrm{e}-7$ & 2.02 & 133.57 & 38.53 \\
\hline
\end{tabular}

\begin{tabular}{|c|c|c|c|c|c|c|c|c|c|}
\hline \multicolumn{10}{|c|}{ Multilevel augmentation schemes when $k=3$} \\
\hline \multirow{2}{*}{$m$} & \multirow{2}{*}{$f(n)$} & \multicolumn{3}{|c|}{ Full multilevel solution } & \multicolumn{3}{|c|}{ Fast multilevel solution } \\
\cline { 3 - 10 } & & $e_{h}^{\prime}$ & $R_{h}^{\prime}$ & $T_{N}^{\prime}$ & $T_{Q}^{\prime}$ & $\widetilde{e}_{h}^{\prime}$ & $\widetilde{R}_{h}^{\prime}$ & $\widetilde{T}_{N}^{\prime}$ & $\widetilde{T}_{Q}^{\prime}$ \\
\hline 0 & 16 & $1.3394 \mathrm{e}-3$ & & 0.16 & 0.10 & $1.3394 \mathrm{e}-3$ & & 0.16 & 0.10 \\
1 & 32 & $2.4112 \mathrm{e}-4$ & 2.47 & 0.38 & 0.25 & $2.4111 \mathrm{e}-4$ & 2.47 & 0.38 & 0.24 \\
2 & 64 & $6.7243 \mathrm{e}-5$ & 1.84 & 0.87 & 0.66 & $6.7237 \mathrm{e}-5$ & 1.84 & 0.74 & 0.53 \\
3 & 128 & $1.5319 \mathrm{e}-5$ & 2.13 & 2.38 & 2.13 & $1.5305 \mathrm{e}-5$ & 2.13 & 1.52 & 1.27 \\
4 & 256 & $3.9538 \mathrm{e}-6$ & 1.95 & 8.28 & 8.13 & $3.9425 \mathrm{e}-6$ & 1.96 & 3.65 & 3.39 \\
5 & 512 & $9.8088 \mathrm{e}-7$ & 2.01 & 34.58 & 34.53 & $9.6895 \mathrm{e}-7$ & 2.02 & 12.61 & 12.43 \\
\hline
\end{tabular}

other nonlinear solvers presented in [2] in regard to their computational complexities.

We note that the multilevel augmentation method discussed in [8] also reduces the overall computational complexity of solving nonlinear Hammerstein equations to $\mathcal{O}(N \log N)$ when no numerical integration is applied. The multilevel augmentation method [8] achieves this computational efficiency by approximating the Jacobian matrices in a subspace of low dimension. In other words, a finite sequence of systems of nonlinear equations is solved at a low and fixed dimension, and corrective terms are appended so that the approximate solutions are optimal in the order of approximation. In this paper, a multilevel augmentation method, presented in section 4, also takes the approach proposed in [8], but in addition the Jacobian matrices were reduced to be sparse. The sparsity was made possible by the fast wavelet collocation method discussed in section 3 . Numerical results show that the fast wavelet collocation method in section 3 and the multilevel augmentation method in section 4 reduce 
the computational time significantly when compared with the standard wavelet collocation method without a truncation strategy. It was observed that the quasi-Newton method provides additional reductions in the computational time for the examples tested for all cases except for the multilevel augmentation methods.

Many results presented in this paper should be extended to nonlinear Hammerstein equations proposed on polygons. This will generalize recent results obtained in [23] and find wider application potentials for the fast wavelet collocation method.

Acknowledgment. We thank the referees for many constructive comments which improved this paper.

\section{REFERENCES}

[1] B. K. Alpert, A class of bases in $L^{2}$ for the sparse representation of integral operators, SIAM J. Math. Anal., 24 (1993), pp. 246-262.

[2] K. E. AtKinson, The Numerical Solution of Integral Equations of the Second Kind, Cambridge University Press, Cambridge, UK, 1997.

[3] K. E. AtKinson, A survey of numerical methods for solving nonlinear integral equations, J. Integral Equations Appl., 4 (1992), pp. 15-46.

[4] K. J. Atkinson, I. Graham, And I. Sloan, Piecewise continuous collocation for integral equations, SIAM J. Numer. Anal., 20 (1983), pp. 172-186.

[5] Z. Chen, C. Micchelli, And Y. Xu, A multilevel method for solving operator equations, J. Math. Anal. Appl., 262 (2001), pp. 688-699.

[6] Z. Chen, C. Micchelli, And Y. Xu, A construction of interpolating wavelets on invariant sets, Math. Comp., 68 (1999), pp. 1569-1587.

[7] Z. Chen, C. A. Micchelli, And Y. Xu, Fast collocation methods for second kind integral equations, SIAM J. Numer. Anal., 40 (2002), pp. 344-375.

[8] Z. Chen, B. Wu, And Y. Xu, Fast multilevel augmentation methods for solving Hammerstein equations, SIAM. J. Numer. Anal., 47 (2009), pp. 2321-2346.

[9] Z. Chen, B. Wu, And Y. Xu, Fast numerical collocation solutions of integral equations, Comm. Pure Appl. Anal., 6 (2007), pp. 643-666.

[10] Z. Chen, B. Wu, AND Y. XU, Multilevel augmentation methods for solving operator equations, Numer. Math. J. Chinese Univ., 14 (2005), pp. 31-55.

[11] W. FANG, F. MA, AND Y. XU, Multi-level iteration methods for solving integral equations of the second kind, J. Integral Equations Appl., 14 (2002), pp. 355-376.

[12] H. Kaneko, R. D. Noren, and B. Novaprateep, Wavelet applications to the Petrov-Galerkin method for Hammerstein equations, Appl. Numer. Math., 45 (2003), pp. 255-273.

[13] H. Kaneko, R. D. Noren, And P. A. Padilla, Superconvergence of the iterated collocation methods for Hammerstein equations, J. Comput. Appl. Math., 80 (1997), pp. 335-349.

[14] H. KANEKo, R. D. NoREn, AND Y. XU, Numerical solutions for weakly singular Hammerstein equations and their superconvergence, J. Integral Equations Appl., 4 (1992), pp. 391-407.

[15] H. Kaneko And Y. Xu, Superconvergence of the iterated Galerkin methods for Hammerstein equations, SIAM J. Numer. Anal., 33 (1996), pp. 1048-1064.

[16] H. KANEKo AND Y. XU, Degenerate kernel method for Hammerstein equations, Math. Comp., 56 (1991), pp. 141-148.

[17] S. KumAR, Superconvergence of a collocation-type method for Hammerstein equations, IMA J. Numer. Anal., 7 (1987), pp. 313-325.

[18] S. Kumar, A discrete collocation-type method for Hammerstein equations, SIAM J. Numer. Anal., 25 (1988), pp. 328-341.

[19] S. Kumar and I. H. Sloan, A new collocation-type method for Hammerstein integral equations, Math. Comp., 48 (1987), pp. 585-593.

[20] C. Micchelli And Y. Xu, Using the matrix refinement equation for the construction of wavelets on invariant sets, Appl. Comput. Harmon. Anal., 1 (1994), pp. 391-401.

[21] C. Micchelli, Y. XU, AND Y. ZhaO, Wavelet Galerkin methods for second-kind integral equations, J. Comput. Appl. Math., 86 (1997), pp. 251-270.

[22] G. VAinikko, A perturbed Galerkin method and the general theory of approximate methods for nonlinear equations, Ž. Vyčisl. Mat. Fiz., 7 (1967), pp. 723-751 (in Russian); U.S.S.R. Comput. Math. and Math. Phys., 7 (1967), pp. 1-41 (in English).

[23] Y. Wang And Y. XU, A fast wavelet collocation method for integral equations on polygons, J. Integral Equations Appl., 17 (2005), pp. 277-330. 